leee Transactions On Geoscience And Remote Sensing

May 2018, Volume 56 Issue 5 Pages 2931-2948

http://dx.doi.org/10.1109/TGRS.2017.2787459

Archimer

http://archimer.ifremer.fr/doc/00440/55156/

(c) 2018 IEEE. Personal use is permitted, but republication/redistribution

requires IEEE permission.

\title{
Modulation of Ka-Band Doppler Radar Signals Backscattered From the Sea Surface
}

\author{
Yurovsky Yury Yu. ${ }^{1}-$, Kudryavtsev Vladimir N. ${ }^{1,2}$, Chapron Bertrand ${ }^{3}$, Grodsky Semyon A. ${ }^{4}$
}

${ }^{1}$ RAS, Marine Hydrophys Inst, Fed State Budget Sci Inst, Sevastopol 299011, Russia.

${ }^{2}$ Russian State Hydrometeorol Univ, Satellite Oceanog Lab, St Petersburg 195196, Russia.

${ }^{3}$ Inst Francais Rech Exploitat Mer, F-29280 Plouzane, France.

${ }^{4}$ Univ Maryland, Dept Atmospher \& Ocean Sci, College Pk, MD 20742 USA.

Corresponding authors : email address : yyyurovsky@gmail.com ; kudr@rshu.ru ;

Bertrand.Chapron@ifremer.fr ; senya@umd.edu

\begin{abstract}
:
This paper presents dual copolarized (VV and $\mathrm{HH}) \mathrm{Ka}$-band radar measurements of joint modulation of normalized radar crass section (NRCS) and Doppler velocity (DV) performed from a sea research platform. NRCS and DV modulations are well correlated. NRCS modulations exhibit a spiky structure. $\mathrm{HH}$ modulations are stronger than VV ones leading to modulations of the polarization ratio. This suggests that an important portion of NRCS modulations is produced by nonpolarized radar returns from modulated wave breaking facets. DV modulations reveal that at incidence angles $<50$ degrees, NRCS spikes are attributable to rather slow moving facets, which may he interpreted as short wave breaking disturbances embedded in the water at crests of modulating waves. Using the DV as a proxy for wave gauge, a modulation transfer function (MTF) is estimated for both polarizations. The hydrodynamics component of the total MTF, hydro-MTF, combines NRCS modulations supported by Bragg waves and wave breaking. The contribution of each type of facets to the hydro-MTF is weighted by its partial contribution to the NRCS, and thus, hydro-MTF becomes dependent on radar polarization. Using hydro-MTF for $\mathrm{HH}$ and VV, Bragg wave and wave breaking modulations are separated. Wave breaking modulations are significant, with MTF amplitude varying from 5 to about 30 depending on wind speed. Ka-band Bragg waves are strongly modulated at low winds, but their modulation almost vanishes at moderate winds. Finally, we propose an empirical MTF parameterization based on polynomial fitting as a function of observation geometry and wind.
\end{abstract}

Keywords: Doppler shift, modulation transfer function (MTF), radar backscattering, sea surface, spikes, wave breaking 


\section{INTRODUCTION}

$\mathbf{O}$ CEAN radar backscattering is modulated by surface waves due to wave-induced modulations of surface slopes (tilt modulation) and roughness (hydrodynamics modulation). Phase modulations of radar backscattering, i.e. Doppler frequency shifts, are governed by local line-of-sight velocity, and thus are also modulated by surface wave orbital velocities. Unresolved, likely modulated, waves with wavelengths shorter than the radar footprint are inherently averaged and contribute

Manuscript received.

The core support of the work was provided by Russian Science Foundation grant No. 15-17-20020. Field experiments in 2014-2015 were supported by FASO of Russia under the State Assignment (No. 0827-2014-0010). At the final stage, this work was partly supported by NASA NNX15AG40G and the Ministry of Science and Education via Goszadanie 5.2928.2017/PP.

Yu.Yu. Yurovsky and V.N. Kudryavtsev are with the Federal State Budget Scientific Institution "Marine Hydrophysical Institute of RAS", Kapitanskaya 2, Sevastopol, Russia, 299011 (e-mail: yyyurovsky@gmail.com).

V.N. Kudryavtsev is also with the Satellite Oceanography Laboratory, Russian State Hydrometeorological University, Malookhtinsky prospect 98, Saint Petersburg, Russia (e-mail: kudr@rshu.ru).

B. Chapron is with the Institut Français de Recherche pour l'Exploitation de la Mer, 29280, Plouzané, France (e-mail: Bertrand.Chapron@ifremer.fr).

S.A. Grodsky is with the University of Maryland, Department of Atmospheric and Oceanic Science, College Park, USA (e-mail: senya@umd.edu). to the time mean normalized radar cross-section (NRCS) and Doppler velocity (DV). Correlated components of DV and NRCS explain the observed C-band Doppler centroid anomalies present even in the absence of currents [1], [2]. Higher frequency radar technique, including $\mathrm{K}_{\mathrm{u}}$-band measurements [3] as well as $\mathrm{K}_{\mathrm{a}}$-band DopplerScat [4] and SKIM [5] build on the combined measurements of NRCS and DV, are aimed to provide new global satellite oceanographic products such as surface currents.

Ocean $\mathrm{K}_{\mathrm{a}}$-band measurements of joint modulation of NRCS and DV are still rare. Early $\mathrm{K}_{\mathrm{a}}$-band measurements have been collected during NORDSEE platform experiments [6], [7]. Impact of waves on radar backscattering modulation is normally described in terms of a complex Modulation Transfer Function (MTF) [8], which experimental estimates are available from tower-based and air/space-borne sensors [6], [7], [9]-[13]. Theoretical explanation of observed MTF is generally based on the relaxation approximation for Bragg waves [14]. But, this approximation results in an unrealistically fast decrease of MTF magnitude with radar frequency. Effect of wind stress modulation by long waves has been proposed to explain the observed magnitude of MTF [12], [15]. However, too strong wind stress modulations are required in order to explain the observations. As shown by [16] and [17], the effect of wave breaking is necessary to explain the time mean and modulation properties of both NRCS and DV.

In this paper, we further report results of multi-year platform-based $\mathrm{K}_{\mathrm{a}}$-band radar measurements. Following the analysis of time mean NRCS [18], the present effort focuses on modulation properties of NRCS and DV. To estimate these properties, we rely on the potential ability of a Doppler radar to act as a wave gauge [8]. To improve this approach, we introduce a correction function associated with radar DV response to LW orbital velocity. Derived dual co-polarized (VV and HH) MTF estimates are fitted by empirical polynomials. Similarly to "Bragg-non-Bragg" decomposition of NRCS [19], the empirical dual co-polarized MTF is decomposed in order to separate the hydrodynamics MTF for Bragg roughness and non-Bragg scatterers (wave breaking).

\section{EXPERIMENT, DATA, AND METHODS}

The measurements were performed from the Black Sea research platform during 2009-2015 using a $37.5 \mathrm{GHz}$ continuous-wave dual co-polarized Doppler $\mathrm{K}_{\mathrm{a}}$-band radar that operated in the hybrid polarization mode with a slant $\left(45^{\circ}\right)$ transmit polarization plane and two receiving channels for vertical and horizontal polarizations. Most of the data and 


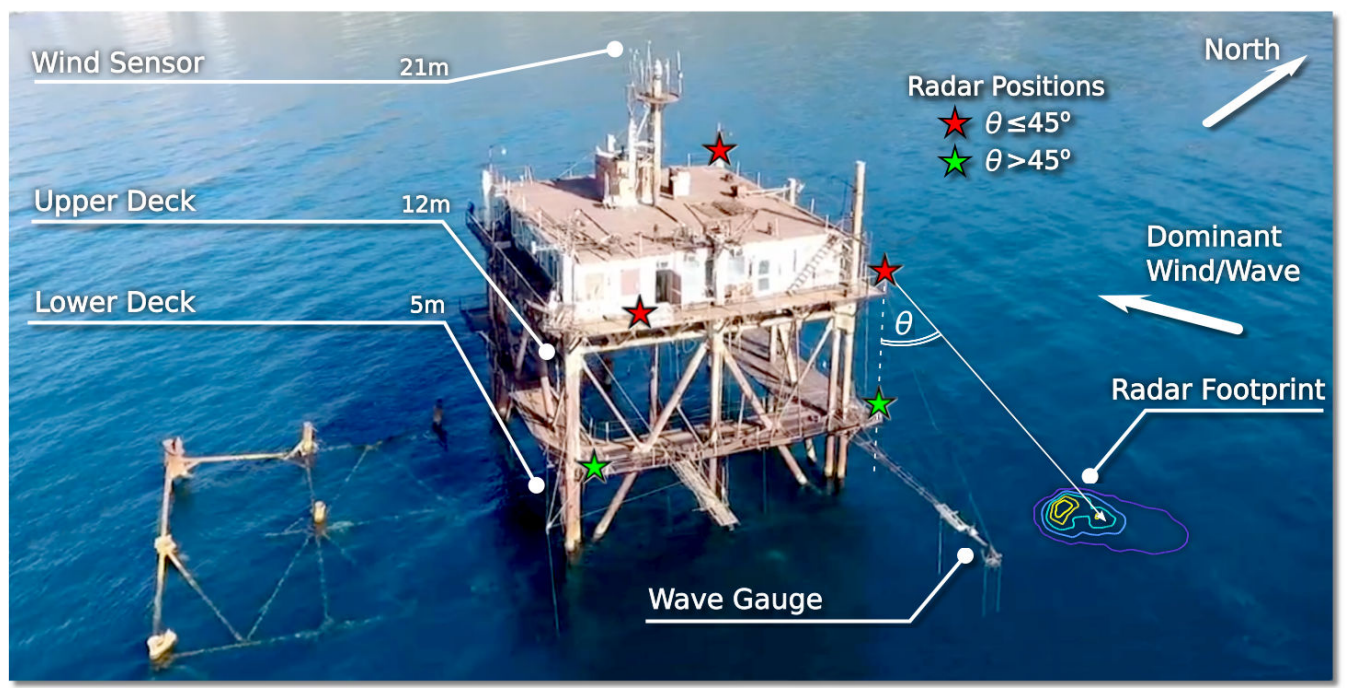

Fig. 1. Black Sea research platform $\left(44^{\circ} 23^{\prime} 35^{\prime \prime} N, 33^{\circ} 59^{\prime} 04^{\prime \prime} E\right)$. Wrecks to the left are remains of another platform destroyed by storms. This south-west azimuth sector is not used for measurements.

instrumentations used in this study are similar to those described in [18]. This configuration implies a cross-polarization contamination of received signals, which, however, is weak for our conditions and is disregarded (see Appendix in [18] for more details).

The radar was installed at different platform locations (heights) depending on desired radar-to-wave azimuth, $\phi$, and incidence angle, $\theta$ (Fig. 1). To keep a reliable balance between radar footprint size, signal-to-noise ratio, and antenna far field applicability distance $(>6 \mathrm{~m})$, the acquisitions were made from either the upper ( $12 \mathrm{~m}$ height $)$ or the lower $(6 \mathrm{~m}$ height $)$ platform decks for $\theta \leq 45^{\circ}$ or $\theta>45^{\circ}$, respectively. The correction for radar antenna pattern is discussed in Appendix A.

Supplementary meteorological measurements were made using the Davis Vantage Pro station with air sensors mounted at $21 \mathrm{~m}$ height. Neutral $10 \mathrm{~m}$ wind speed, $U$, was calculated using the COARE3.0 algorithm [20]. Surface wave elevations were recorded by six wire wave gauge antenna ( 5 strings in corners of a pentagon and one in its center, each pair separated by about $25 \mathrm{~cm}$ ) installed from $11 \mathrm{~m}$ horizontal stern. The distance between wave gauge and radar footprint varied from a few meters to tens of meters, which did not allow for phase comparison between radar and wave elevation signals.

Directional surface wave spectra were estimated over 30 min intervals by the maximum likelihood method (DIWASP package [21]), from which the wave peak frequency, $f_{\mathrm{p}}$, and its direction, $\phi_{\mathrm{p}}$, were estimated. Complex situations with short offshore fetches, strong swell, and multiple swell peaks were eliminated from the analysis. Only records with co-aligned wind and waves were included while records with wind and wave directions deviating by $>30^{\circ}$ were not included. For the included records, radar-to-wind and radar-to-wave azimuths were assumed equal (radar-to-wave azimuth was used by default). Throughout the text, the following azimuth notations are used: $\phi=0^{\circ}-$ upwind, $\phi=90^{\circ}-$ crosswind , $\phi=180^{\circ}$ - downwind.
Doppler measurements performed from a static platform allow to detect both the instantaneous (centroid of the instantaneous Doppler spectrum) and the NRCS-weighted (centroid of the time-mean Doppler spectrum) Doppler shifts, and thus distinguish between the mean scatterer velocity and the waveinduced contribution.

The raw radar data processing followed [22]. In-phase, $I(t)$, quadrature, $Q(t)$, signal components were digitized at $40 \mathrm{kHz}$ rate for $\mathrm{VV}$ and $\mathrm{HH}$ polarizations, from which instantaneous Doppler spectra were computed over consecutive $\tau=0.2 \mathrm{~s}$ intervals,

$$
S(\nu, t)=\overline{\left|\int_{t-\tau / 2}^{t+\tau / 2}(I+i Q) e^{-i 2 \pi \nu t} \mathrm{~d} t\right|^{2}},
$$

where $\overline{(\ldots)}$ stands for ensemble averaging with 2 degrees of freedom, $\nu$ is the Doppler frequency. Instantaneous NRCS, $\sigma(t)=\int S(\nu, t) \mathrm{d} \nu$, Doppler frequency shift, $f_{\mathrm{D}}(t)=$ $\int \nu S(\nu, t) \mathrm{d} \nu / \sigma(t)$, and Doppler bandwidth (DW), $w_{\mathrm{D}}(t)=$ $\int\left(\nu-f_{\mathrm{D}}(t)\right)^{2} S(\nu, t) \mathrm{d} \nu / \sigma(t)$, were estimated as corresponding momenta of the instantaneous Doppler spectrum, $S(t, \nu)$. Instantaneous Doppler frequency shift, $f_{\mathrm{D}}(t)$, translates into instantaneous line-of-sight (LOS) Doppler velocity, $v(t)=$ $\pi k_{\mathrm{r}}^{-1} f_{\mathrm{D}}(t)$, where $k_{\mathrm{r}}$ is the radar wavenumber.

The original radar records were sub-divided into $5 \mathrm{~min}$ samples. The total number of analyzed samples is about 500. Data distribution versus incidence angle, $\theta$, radar-to-wave azimuth, $\phi$, and wind speed, $U$, is shown in Fig. 2 . The absence of data at low winds $(U<3-4 \mathrm{~m} / \mathrm{s})$ is due to low signalto-noise ratio under these conditions. The majority of the data are collected at moderate winds, $U=8-12 \mathrm{~m} / \mathrm{s}$. Downwind radar orientations are rare because of platform shadowing.

\section{Radar Signal Features}

\section{A. Time Series}

Time series of running VV and HH Doppler spectra (computed over consecutive $0.2 \mathrm{~s}$ intervals, Fig. 3) show a marked 

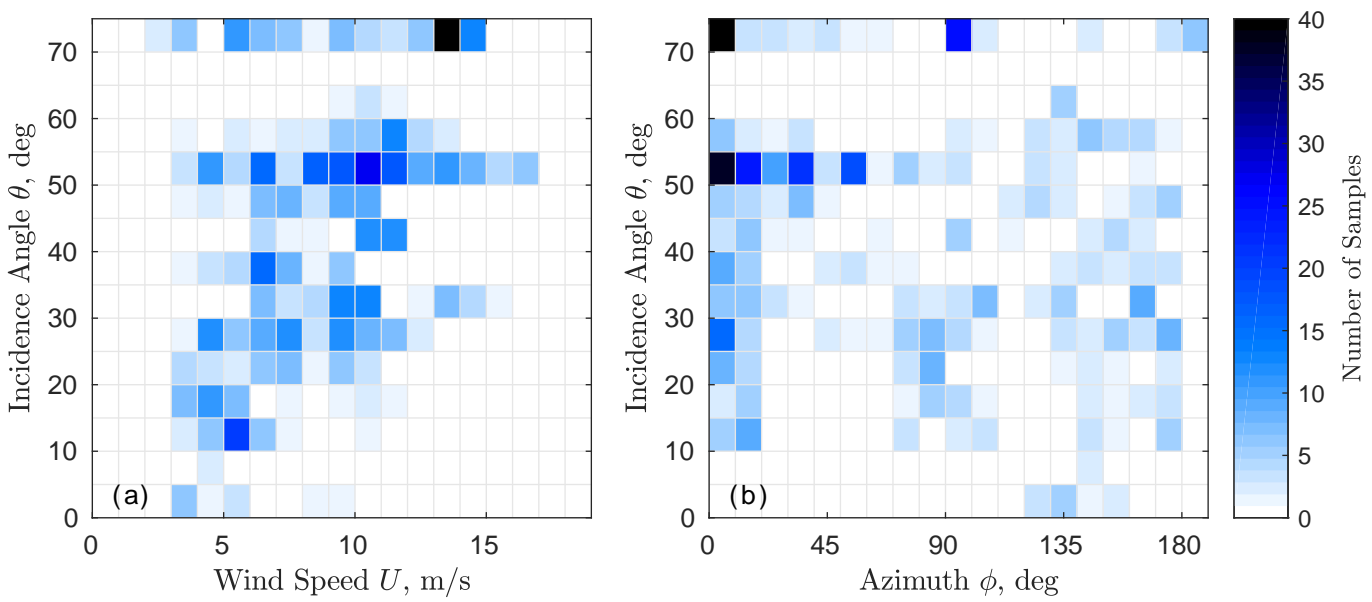

Fig. 2. Dataset statistics: number of data samples versus (a) incidence angle, $\theta$, and wind speed, $U$, and (b) incidence angle, $\theta$, and radar-to-wind/wave azimuth, $\phi$.

modulation certainly associated with long surface waves with a period of about $4 \mathrm{~s}$. The spatial scale resolved by a radar is determined by its ground footprint size. The latter depends on the incidence angle and in our case may vary by a few meters (see Appendix A for more details). We refer to these resolved waves as Long Waves (LW), hereinafter.

Fig. 3 reveals the occurrence of radar signal spikes also linked to local maxima of the Doppler frequency shift. From visual observations, these spikes are usually associated with breaker passages within the radar footprint. Note also that NRCS spikes are not accompanied by noticeable DV spikes, at least at $\theta \approx 53^{\circ}$ and $U=10 \mathrm{~m} / \mathrm{s}$. This suggests that measured NRCS spikes do not necessarily correspond to "fast moving" scatterers, but rather slow moving very rough surface elements with the translation speed somewhat higher than the LW orbital velocity. Fig. 3 also shows that Doppler spectrum bandwidth (DW) correlates well with the DV. This indicates that the spread of radar scatterer velocities increases in areas where the DV has local maxima (wave crests for upwind observation geometry in Fig. 3). In other words, the scatterers in rough areas associated with breaking waves do not move as "frozen" targets, but have rather wide speed distribution.

As was first suggested in [8], the Doppler signal can be used for direct estimation of LW orbital velocities, and thus, after integration, LW surface displacements and tilts. Assuming that DV fluctuations are solely produced by LW orbital velocities, the relationship between Fourier harmonics of wave elevation, $\hat{\xi}$, and DV, $\hat{v}$, can be written as [8], [23]:

$$
\begin{aligned}
\hat{v}(\omega) & =\omega G(\theta, \phi) \hat{\xi}(\omega), \\
G(\theta, \phi) & =\cos \phi \sin \theta+i \cos \theta,
\end{aligned}
$$

where $\omega$ is the LW frequency, $\theta$ is the incidence angle, $\phi$ is the radar-to-wave azimuth, $G$ is the geometric transformation coefficient projecting the wave orbital velocity onto the radar LOS direction, and hat above any quantity denotes its Fourier transform. In this study, we ignore the shallow water effects. During the experiments, LW wave numbers were in the range $k>0.1 \mathrm{rad} / \mathrm{m}$, that for water depth $D=25 \mathrm{~m}$ gives $k D>$ 2.5 .

NRCS modulations by LWs are described in terms of the radar Modulation Transfer Function (MTF):

$$
M=\frac{\hat{\sigma}}{\bar{\sigma} k \hat{\xi}},
$$

where $k$ is the LW wave number linked to $\omega$ by the deep water dispersion relationship, $\omega^{2}=g k, g$ is the gravity acceleration, $\bar{\sigma}$ is the mean (averaged over LW phase) NRCS, $\hat{\sigma}$ is the Fourier transform of NRCS modulations by the LW with amplitude, $\xi$. By definition, the MTF describes a linear relationship between wave slopes $(k \hat{\xi})$ and relative NRCS variations $(\hat{\sigma} / \bar{\sigma})$, which must be small. Although in our case the latter requirement is not always the case, we accept the linear MTF concept for comparability with the previous studies, (e.g. [6], [8], [23], and others).

Using (2) and (3), the MTF (4) can be calculated from Doppler radar measurements as:

$$
M=\frac{g G S_{\sigma v}}{\bar{\sigma} \omega S_{v v}}
$$

where $S_{\sigma v}$ is the cross-spectrum between NRCS and DV time series, and $S_{v v}$ is the auto-spectrum of DV time series.

Besides dependencies on LW tilt and roughness modulations, platform-based NRCS also depends on wave-induced changes in range and footprint area. For the beam-limited footprint, the range dependence is proportional to $R^{-2}$ (see e.g. eq.(45) in [23]), while the footprint area varies as $\cos (k \xi)$. The range and footprint area modulations were removed from the received power using instantaneous range and LW tilt modulations derived from the measured DV, eqs. $(2,3)$.

Sample time series of NRCS, polarization ratio, PR = $\sigma^{\mathrm{vv}} / \sigma^{\mathrm{hh}}$, and DV are shown in Fig. 4 together with LW elevation, $\xi$, and slope, $\zeta$, computed from VV DV using eqs. $(2,3)$. Both, VV and HH NRCS are well correlated with LW elevations and slopes derived from DV measurements. At low incidence angles, $\theta=15^{\circ}$ and $35^{\circ}$, the NRCS is enhanced 


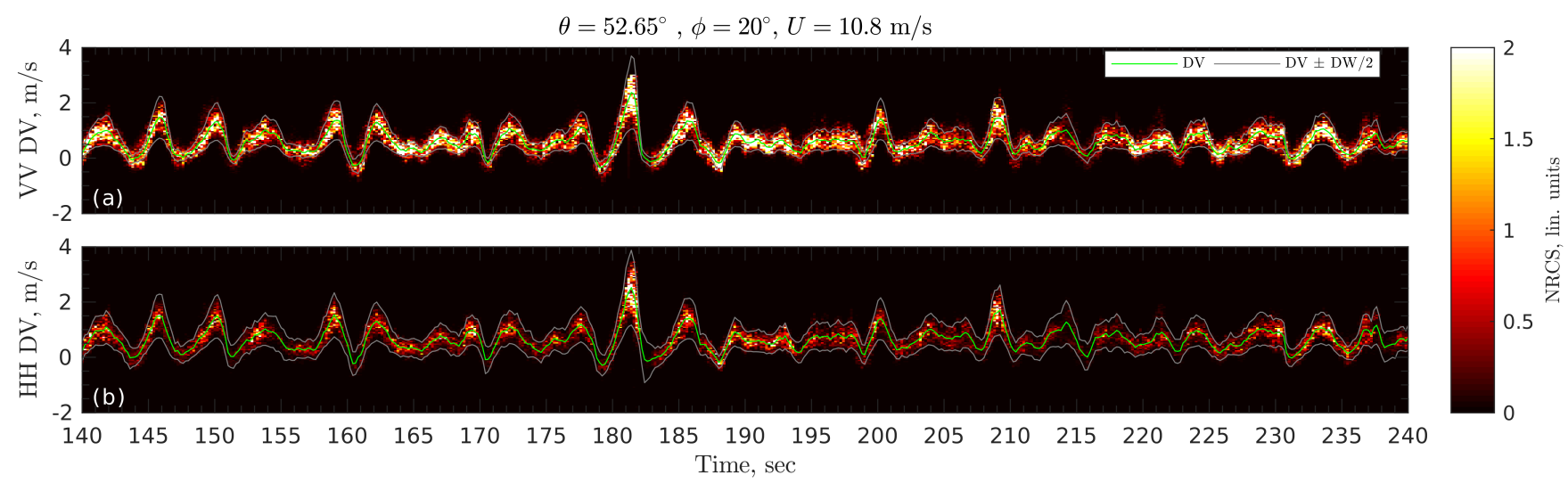

Fig. 3. An example of instantaneous (a) VV and (b) HH Doppler spectra $\left(\theta \approx 53^{\circ}\right.$, upwind, $\left.U=10 \mathrm{~m} / \mathrm{s}\right)$ as a function of time. Doppler velocity (green, middle line) and Doppler spectrum width (gray, top and bottom lines) are shown. Color shades correspond to spectrum density.

on forward LW slopes suggesting the dominant role of the tilt modulation mechanism. At larger $\theta$, the relative importance of the tilt modulation decreases and the maximum of NRCS modulation shifts towards LW crests and approximately follows wave elevations. This reflects a major contribution of the hydrodynamics modulation to the total MTF.

The measured PR is systematically lower than the PR predicted by a standard two-scale Bragg model (TSM, see Appendix B for its evaluation details). A remarkable feature in Fig. 4 is an apparent correlation between strong NRCS peaks and PR drops. For some of large NRCS peaks, the PR drops down to $0 \mathrm{~dB}$ indicating that NRCS peaks are non-polarized, $\sigma^{\mathrm{vv}}=\sigma^{\mathrm{hh}}$. Enhanced NRCS peaks presumably represent nonpolarized radar returns from steep and rough surface patterns related to breaking waves (see, e.g., Figs. $4 \mathrm{~b}$ at $t=62 \mathrm{~s}$, $4 \mathrm{c}$ at $t=82 \mathrm{~s}, 4 \mathrm{~d}$ at $t=87 \mathrm{~s})$. Excepting cases of $\mathrm{HH}$ backscattering at high incidence angles $\left(\theta=70^{\circ}\right)$, the DV exhibits quite smooth behavior with modulations comparable with LW orbital velocities (see also Fig. 7 below). A notable feature is that strong NRCS peaks are not accompanied by remarkable DV "spikes". This suggests that, at our observation geometry, wave breaking enhancement on crests of LWs does not lead to DV anomalies apparently deviating from LW orbital velocities.

\section{B. Distributions, Skewness and Doppler Spectra}

Scatter plots "NRCS versus DV" and "PR versus DV" corresponding to the time series in Fig. 4 are presented in the left and middle columns of Fig. 5. The most apparent feature is the positive correlation between DV and NRCS variations reflecting NRCS enhancement on forward slopes close to wave crests where DV has maximum positive values for the upwind observation geometry. At $\theta>50^{\circ}$, PR and DV variations are negatively correlated (Fig. 5g,j) suggesting that NP backscattering from modulated breaking waves does contribute at these incidence angles.

The centered probability density functions (PDF, Fig. 5, right column) provide a deeper insight into specific features of joint modulations of NRCS and DV. First, the distribution of DV is quite symmetrical relative to its mean value, while the distribution of either VV or HH NRCS as well as PR (all plotted in logarithmic scale) is strongly skewed (if converted to linear scale). This shows that enhancements of NRCS in areas of positive DV (wave crests for upwind observations) are much stronger than its suppressions in areas of negative DV. The skewness of NRCS, PR, and DV PDFs becomes more pronounced at large $\theta$ (Fig. 5i,l). Interestingly, that distribution of DV and $\log$ (NRCS) is inversely skewed for this particular observation geometry. Doppler velocity PDF has a positive "tail" corresponding to fast breakers moving towards an upwind looking radar. The negative "tail" of $\log (\mathrm{NRCS})$ PDF is explained by signal drops (pauses) over turbulent patches following wave breaking events.

Doppler spectra (Fig. 5, right column) exhibit features similar to those present in DV PDFs. In particular, the tail corresponding to positive DVs becomes more pronounced at large $\theta$. This is better seen in HH Doppler spectrum at $\theta=70^{\circ}$ (Fig. 51), which has a rather broad peak area extending into positive DVs of about $2.5 \mathrm{~m} / \mathrm{s}$. This is in line with previous observations (e.g. [24]-[26]) that have been attributed to "fast scatterers" associated with breaking waves. The similarity between DV PDFs and Doppler spectra allows using the skewness of DV PDF as a measure of Doppler spectrum asymmetry and corresponding fast scatterer impact.

The skewness characteristics for different observation geometries are shown in Fig. 6. The $\log$ (NRCS) skewness is near zero at $\theta \approx 20^{\circ}$ and grows rapidly with increasing $\theta$ from $\approx-0.5$ near the nadir to $\approx 0.5$ at $\theta=70^{\circ}$. Interestingly, this growth is not regular. There is a local maximum at $\theta=30-35^{\circ}$ which is more pronounced for $\mathrm{VV}$ polarization. This maximum can be associated with a local maximum in the gradient of the tilt MTF, $\partial^{2} \sigma / \partial \theta^{2}$, that leads to nonlinear NRCS modulation. At $\theta>55^{\circ}$, the skewness grows again due to the contribution of strong positive spikes from wave breaking that is more prominent at $\mathrm{HH}$ than at $\mathrm{VV}$ polarization. The skewness of DV increases in magnitude with the incidence angle. Its sign is azimuth dependent reflecting the relative direction of fast moving breakers. 
(a) $\quad \theta=15^{\circ}, \phi=1^{\circ}, U=9.7 \mathrm{~m} / \mathrm{s}$

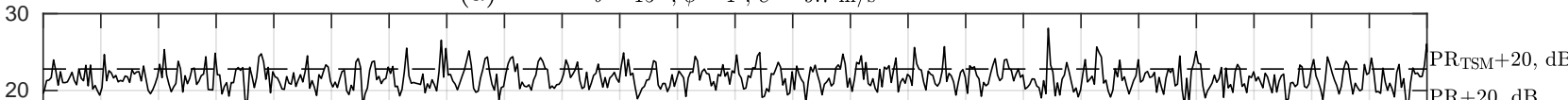

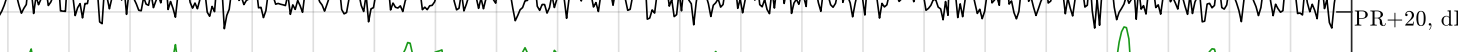

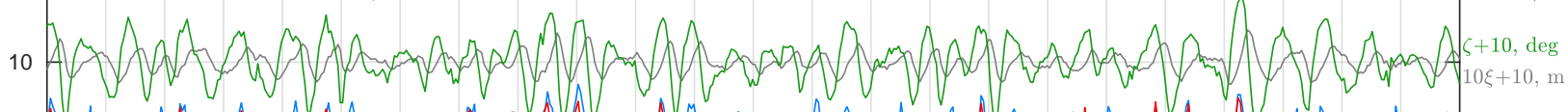

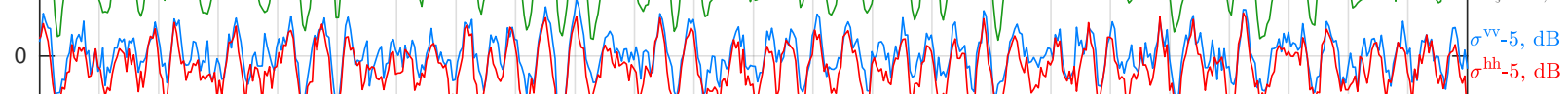
Ww

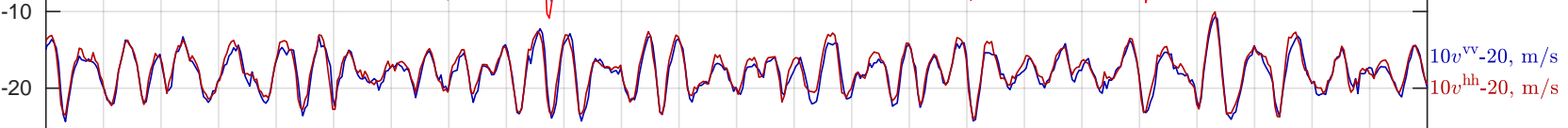

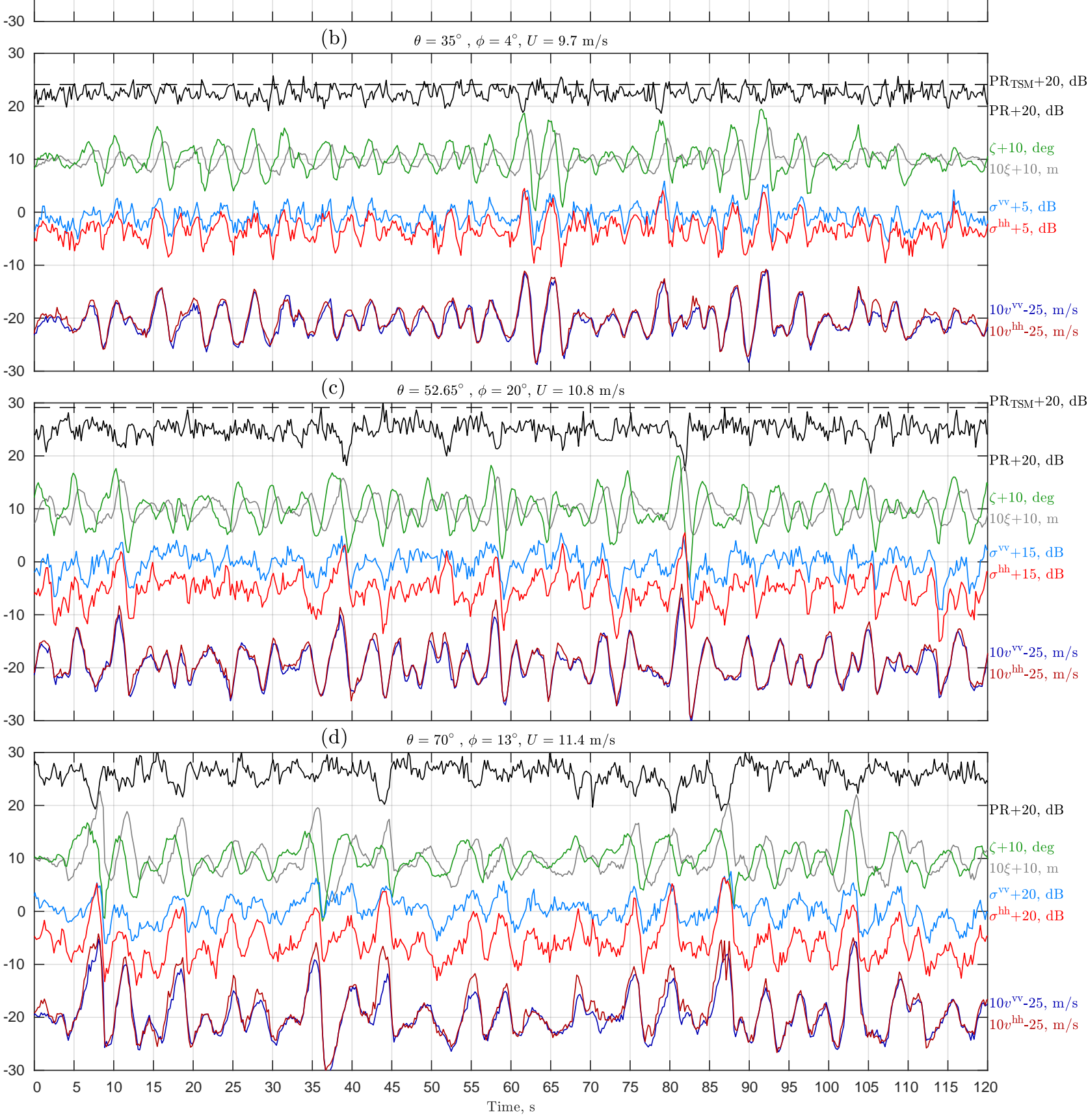

Fig. 4. Time series of normalized radar cross-section $\left(\sigma^{\mathrm{vv}}-\right.$ red, $\sigma^{\mathrm{hh}}-$ blue $)$, Doppler velocity $\left(v^{\mathrm{vv}}-\right.$ dark red, $v^{\mathrm{hh}}-$ dark blue), polarization ratio (PR, solid black), wave elevation ( $\xi$ - gray) and wave slope $(\zeta-$ green) inferred from VV DV. Theoretical two-scale model (TSM) PR is shown by horizontal black dashed lines (for $\theta=70^{\circ}$ TSM PR is out of scale). The signals are shifted along the $y$-axis for better visibility. 

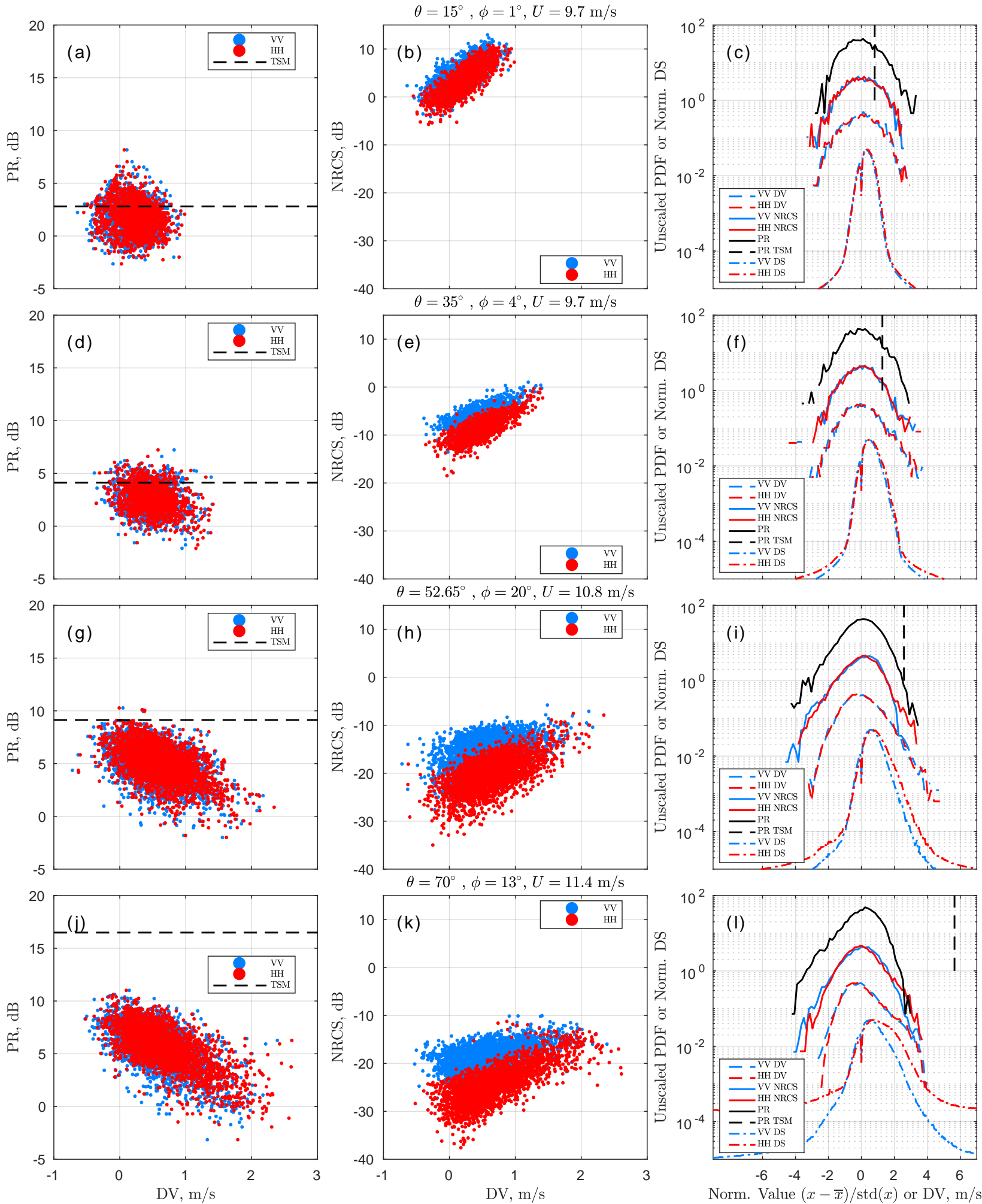

Fig. 5. Scatter plots of (left) instantaneous PR vs DV, (middle) NRCS vs DV. Right column presents unscaled probability density functions of centered PR, NRCS, DV, and unscaled Doppler spectra (DS, the $x$-axis for Doppler spectra is DV in $\mathrm{m} / \mathrm{s}$ ). The rows correspond to the time series shown in Fig. 4. 

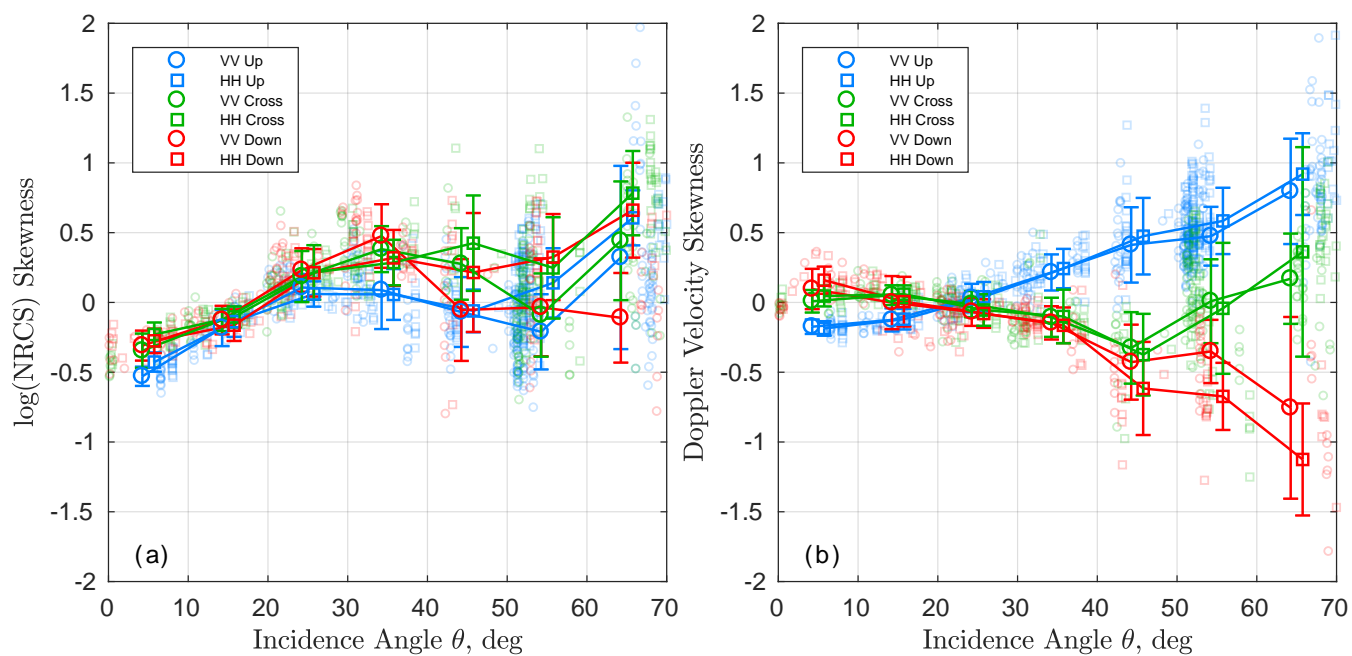

Fig. 6. Skewness of instantaneous (a) NRCS in logarithmic units, (b) Doppler velocity, and (c) Doppler acceleration (circles are VV, squares are HH, blue is upwind, green is crosswind, red is downwind) versus incidence angle for all conditions.

\section{RELATION OF DV OsCillations to LW ORbital Motions AND WaVE BREAKING Modulations}

\section{A. DV-Derived Elevation Spectra}

When DV is used as a proxy for wave gauge, it is usually assumed that DV oscillations are produced solely by the orbital motions [23] while other mechanisms of DV modulations (e.g. by wave breaking) are disregarded. To account for these latter mechanisms, a DV response function defined as the ratio between the measured DV spectrum and its expected value from the linear wave theory is introduced:

$$
\mu=\frac{S_{v v}}{|G|^{2} \omega^{2} S_{\xi \xi}}
$$

where $S_{v v}$ is the DV spectrum corrected for the effect of footprint filtering (see eq. (20) in Appendix A).

Using this definition, the DV variation (2) is modified as:

$$
\hat{v}=\mu^{1 / 2} G \omega \hat{\xi} \text {. }
$$

The spectral shape of the response function, $\mu$, is evaluated from wave gauge measurements (Fig. 7). The latter is neither contemporaneous in time nor collocated in space with the radar time series. Therefore, only auto spectra of DV and LW parameters can be jointly analyzed.

Typical wave gauge frequency elevation spectra and elevation spectra derived from DV measurements,

$$
S_{\xi \xi}=|G|^{-2} \omega^{-2} S_{v v},
$$

demonstrate rather good agreement especially in the vicinity of their spectral peaks. As already discussed, such agreement is not expected given the significant impact of wave breaking modulations on radar signal modulations (Figs. 4, 5). But, no remarkable difference between DV spectrum level and that derived from wave gauge data can be revealed in the spectrum peak area at low to moderate $\theta$ (Fig. 7a,b,c). A noticeable excess appears only at large incidence angles, $\theta=70^{\circ}$, and HH polarization (Fig. 7d).
The DV response function is close to 1 between the wave peak frequency, $f_{\mathrm{p}}$, and the radar pattern cut-off frequency, $f_{\text {cut }}$. At $f>f_{\text {cut }}$, its magnitude rapidly increases while DVNRCS coherence almost vanishes (see Appendix A for details on $f_{\text {cut }}$ estimation). Below $f_{\mathrm{p}}$, the DV response function is also increases indicating non-negligible low frequency DV fluctuations probably caused either by wave groups, wind speed variations, or other modulation mechanisms which examination is out of the scope of this paper.

Assuming that $\mu$ is frequency independent, it is averaged over $f_{\mathrm{p}}<f<f_{\text {cut }}$. For each data sample, the resulting averaged DV response magnitude is used to correct the orbital velocity auto-spectrum $\left(S_{v v}\right.$ in eq. (9)). The mean DV response magnitude (Fig. 8) also illuminates the role of DV spikes associated with fast scatterers. One can note that $\mu$ increases in the crosswind direction. Probably, this increase results from uncertainties in wave azimuth estimation. In the crosswind azimuth sector, even small errors in $|G|^{2}$-factor in the denominator of eq. (6) leads to rather large errors in $\mu$, especially at large $\theta$. On the other hand, the magnitude of $\mu$ also increases at large $\theta \approx 70^{\circ}$ (stronger for $\mathrm{HH}$ polarization than for VV polarization). This agrees with the concept of fast scatterers (wave breaking) that dominate $\mathrm{HH}$ return at large incidence angles.

\section{B. Are the Breakers Fast or Slow Scatterers?}

As already noticed, the remarkable deviation of observed PRs from Bragg-model values and the negative correlation between DV and PR (see Fig.5, left column) suggest that NRCS peaks appearing on LW crests are associated with NP radar returns from breaking waves. In addition, a reasonably good correspondence between DV-derived and wave gauge elevation spectra suggests that breaking waves are (on average) "slow" facets because the measured DV is not "distinguishable" from the LW orbital velocity (except for $\mathrm{HH}$ at $\theta=70^{\circ}$ ). Thus, at first glance, our observations conflict with a number of 


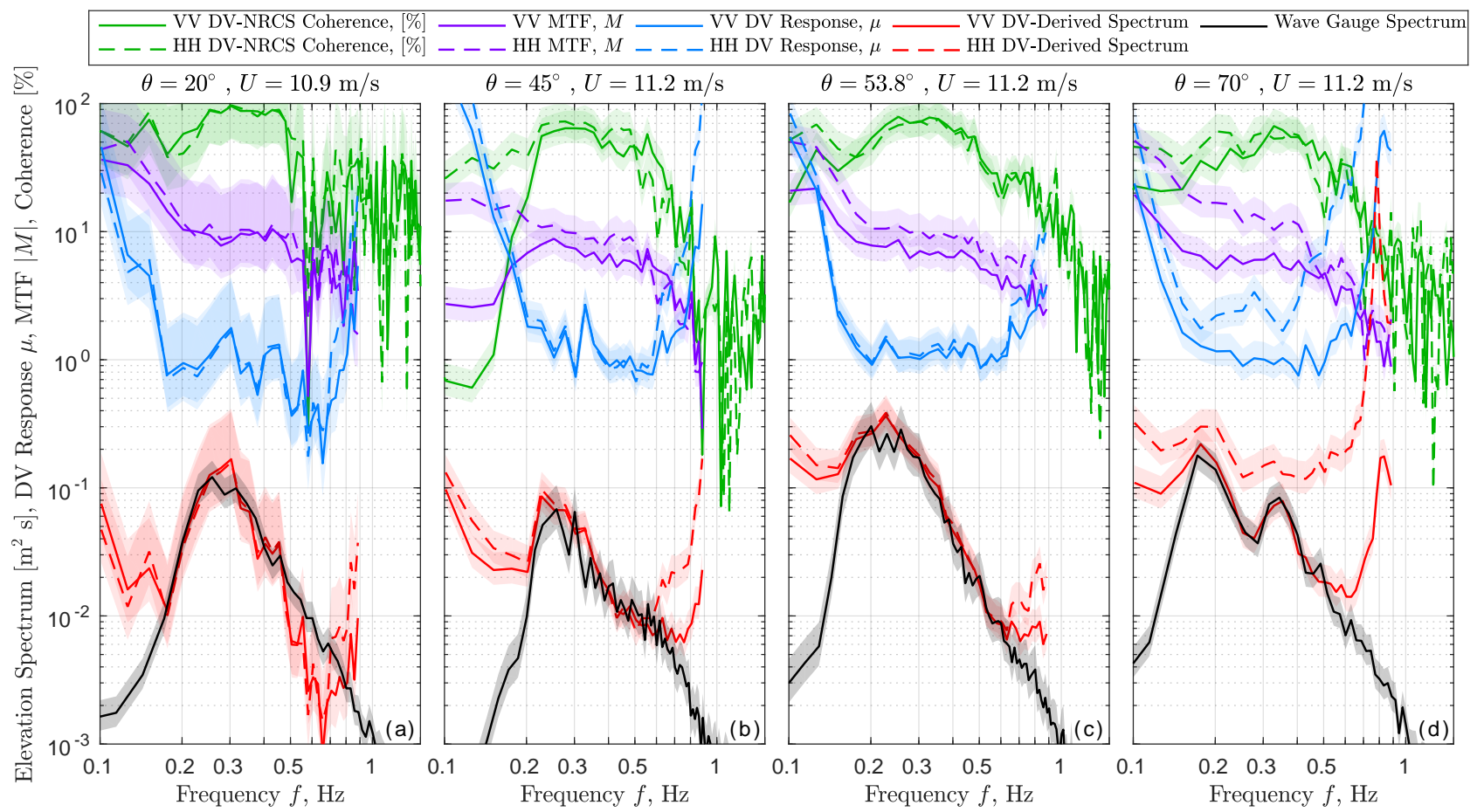

Fig. 7. Upwind coherence between DV and NRCS, MTF $(M)$, DV response function $(\mu)$, DV-derived elevation spectra, wave gauge elevation spectra for (a) $\theta=20^{\circ}$, (b) $\theta=45^{\circ}$, (c) $\theta=53.8^{\circ}$, and (d) $\theta=70^{\circ}$. DV-derived spectra are corrected for the radar footprint response function, eq. (20).

previous studies suggesting that wave breaking are fast moving facets. A few examples follow.

At low grazing angles, Seeman et al. [27] have observed strong X-band DV spikes (up to $7 \mathrm{~m} / \mathrm{s}$ ) corresponding to the phase speed of shoaling breaking wave (see their Fig. 4). In wave tank experiments with different microwave bands (from the S- to the W-band), Lamont-Smith et al. [28] have found that DV of breakers produced by wave focusing corresponds well to their phase velocity (see their Fig. 4).

But in the open sea conditions, the DV of radar spikes is normally lower than the spectrum peak phase speed. In particular, the DV of $\mathrm{K}_{\mathrm{u}}$-band spikes observed by Jessup et al. [29] at $\theta=45^{\circ}$ constituted only $\approx 25 \%$ of the spectral peak phase speed. Similarly low DV values were reported in [24], [26] in the X-band and in [30] in the S-band at low grazing angles. Real breaking waves are distributed over a wide range of scales extending from the spectrum peak of wind generated waves to gravity waves with wavelengths of the order of $1 \mathrm{~m}$. The shorter the breaking waves are the larger the surface probability breaker density produced by these waves is [31]. LWs strongly modulate breaking of shorter waves [32], therefore the crests of LWs (i.e. spectrum peak waves) are covered by short-scale breakers whose DV is lower than the phase speed of modulating LWs. This fact may explain the difference between DVs measured in the laboratory and open ocean conditions [33].

Nevertheless, if the DV of a short-scale breaker is equivalent to its phase velocity, a remarkable contribution of modulated breaking waves to LW-induced DV oscillations could be expected. In our measurements, such contribution is noticeable at large incidence angles and $\mathrm{HH}$ polarization, but it is not noticeable at smaller incidence angles (compare Fig. $7 d$ with other panels in Fig. 7). This observation suggests that DV of radar backscattering from "individual" breakers, besides LOS factor, depends on the incidence angle.

The following paradigm could justify this incidence angle dependence. A breaker originates from the explosive crest instability leading to the rapid overturning of the surface (beginning of breaker generation). Then, during its life span, an active breaker advances with the phase velocity of its carrying breaking wave, and leaves behind an expanding area of surface disturbances (enhanced surface roughness). Most likely, these disturbances are "embedded" into the water, and thus their DVs correspond to the "water velocity". In contrast, the DV of radar backscattering from the forward slope of an active breaker corresponds to its advance velocity, and thus is close to the phase velocity of carrying breaking wave. Thus, at large incidence angles and in the upwind direction, a radar detects breakers as fast facets. At moderate incidence angles, most of the radar backscattering is produced by the enhanced roughness on the top of breakers (due to larger than forward face area), which are thus detected as slow facets.

Such paradigm may explain the observed Doppler features in a wide range of incidence angles. At any incidence angle, the incoherent NRCS of "individual" breakers is much higher than the ambient NRCS produced by resonant Bragg scattering. As a result of such NP contribution, the NRCS peaks and the PR drops on LW crests where wave breaking intensifies (in line with Fig. 4 and Fig.5, scatter plots). At large incidence angles and in the upwind direction, forward 

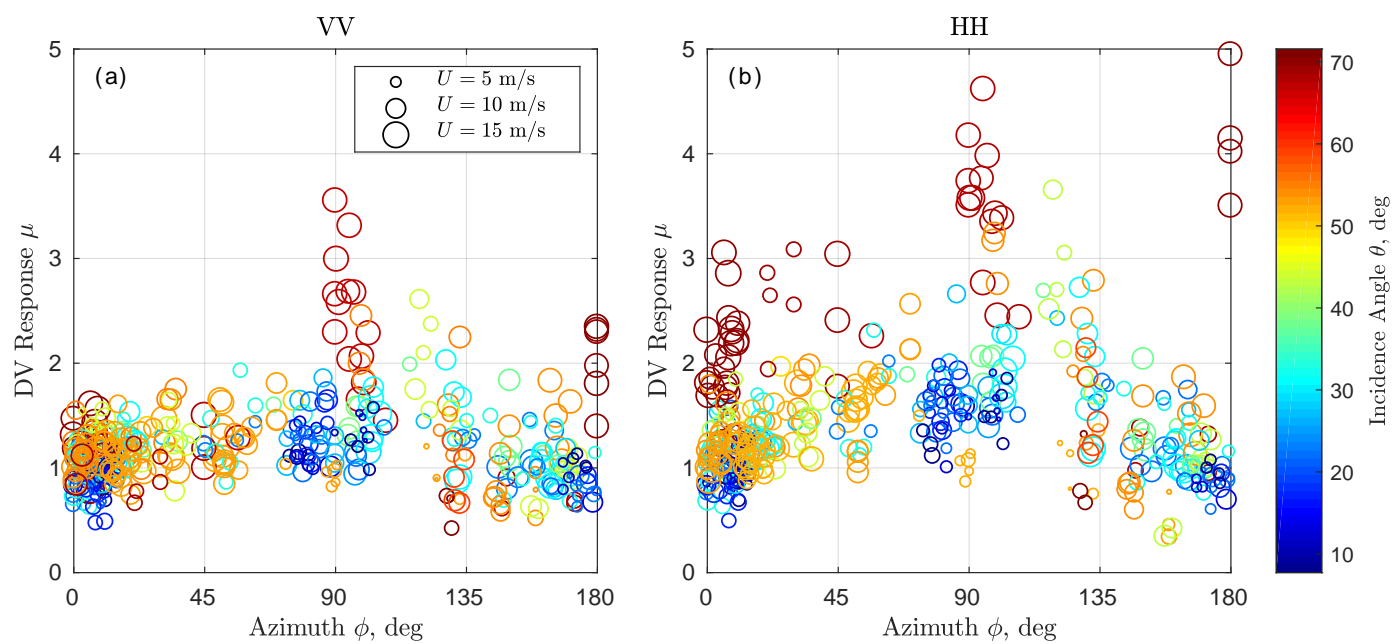

Fig. 8. DV response function, $\mu$, averaged over wave frequency range $f_{\mathrm{p}}<f<f_{\text {cut }}$ as a function of azimuth for (a) VV and (b) HH polarization. Colors correspond to incidence angle, symbol size is proportional to wind speed.

faces of active breakers are exposed. Hence, NRCS peaks are formed by the fast scatters that leads to strong asymmetry of Doppler spectra and the deviation between DV-derived and wave gauge elevation spectra (Fig. 51 and Fig. 7d). In contrast, at moderate incidence angles, NRCS spikes correspond to the slow facets (embedded in the water), which lead neither to the significant asymmetry of Doppler spectra (Fig. 5f and Fig. 5i) nor the apparent deviation between DV-derived and wave gauge spectra (see Fig. 7b and Fig. 7c). Note also that this paradigm and the data do not support the Hansen et al. [34] assumption that DV of an "individual" breaker corresponds to the phase velocity of carrying wave at any incidence angle.

\section{RADAR MTF}

Wave-induced DV variations are used to assess the radar MTF (5). To take into account possible deviations of DV from LW orbital velocities, the DV response function, $\mu$, defined by (6) is included in the radar MTF estimate (5) as:

$$
M=\mu^{1 / 2} \frac{g G S_{\sigma v}}{\bar{\sigma} \omega S_{v v}} .
$$

Examples of estimated MTF's are shown in Fig. 7. Swell cases (e.g. cases like Fig. 7d), as well as cases with low coherence $(<0.2)$ were excluded from our analysis. Spectral dependency of MTF in the frequency range of resolved wind waves from $f_{\mathrm{p}} \approx 0.2 \mathrm{~Hz}$ to $f_{\text {cut }}=0.3-0.8 \mathrm{~Hz}$ (that depends on the look geometry, see Appendix A for details) is not accounted for. Instead, the mean MTF averaged over $f_{\mathrm{p}}<f<f_{\text {cut }}$ is used for each data sample. The mean MTF is fitted by empirical polynomials of incidence angle, azimuth, and wind speed (see Appendix $\mathrm{C}$ for details).

MTF magnitude (Fig. 9a-f) has a local maximum around $\theta=20^{\circ}$ and a local minimum around $\theta=50^{\circ}$. A plausible explanation is related to the tilt MTF which has a maximum around $\theta=20^{\circ}$ (where radar scattering switches from the specular to the resonant mechanism), see e.g. Fig. 11 below. Due to modulation of surface density of breakers by LW, the
MTF magnitude increases at $\theta>50^{\circ}$ (with a stronger impact on $\mathrm{HH}$ ), which may explain the local MTF minimum around $50^{\circ}$. This is not the case for light winds and VV polarization (Fig. 9a) for which Bragg backscattering dominates over breaking backscattering.

As expected from the previous studies [6], [16], VV MTF magnitude is generally smaller than HH MTF magnitude that is likely attributed to stronger tilt modulation at $\mathrm{HH}$ polarization. At $20^{\circ}<\theta<50^{\circ}$, the MTF phase is approximately independent of incidence angle with NRCS modulation maxima locating on the front/rear LW slope for the upwind/downwind radar azimuth, respectively.

Crosswind MTF phase switches sharply from positive to negative angles at $\theta=60^{\circ}$. Noting that tilt MTF zeros in the crosswind direction and disregarding possible errors in radar-to-wind/wave azimuth, such behavior indicates that backscattering peak moves from rear wave slopes at $\theta<60^{\circ}$ to front wave slopes at $\theta>60^{\circ}$.

To the best of our knowledge, there are two publications on analysis of $\mathrm{K}_{\mathrm{a}}$-band MTF; they are both based on measurements taken from the NORDSEE platform located in 30 $\mathrm{m}$ deep water, similar to the Black Sea platform. Feindt et al. [6] reported $\mathrm{K}_{\mathrm{a}}$-band measurements from $30.5 \mathrm{~m}$ height at $\theta=60^{\circ}$, mostly upwind azimuths, and presented radar MTF values averaged over two frequency ranges $(0.11<$ $f<0.14 \mathrm{~Hz}$ corresponding to swell and $0.23<f<0.26$ $\mathrm{Hz}$ corresponding to wind sea). Keller et al. [7] data were collected at $\theta=45^{\circ}$ at different radar heights and radarwind azimuths (in the upwind to crosswind sector). Reported MTF represents the mean values averaged over wind wave frequencies $(0.25<f<0.3125 \mathrm{~Hz})$.

Wind dependence of our MTF magnitude (Fig. 10a,b) is in good agreement with Keller et al. [7] for VV polarization except at $U<4 \mathrm{~m} / \mathrm{s}$. For HH polarization, our MTF is higher at $U<9 \mathrm{~m} / \mathrm{s}$. Feindt et al. [6] data averaged over the wind wave frequency range (similar to our resolved frequency range) are almost two times lower for both polarizations. 

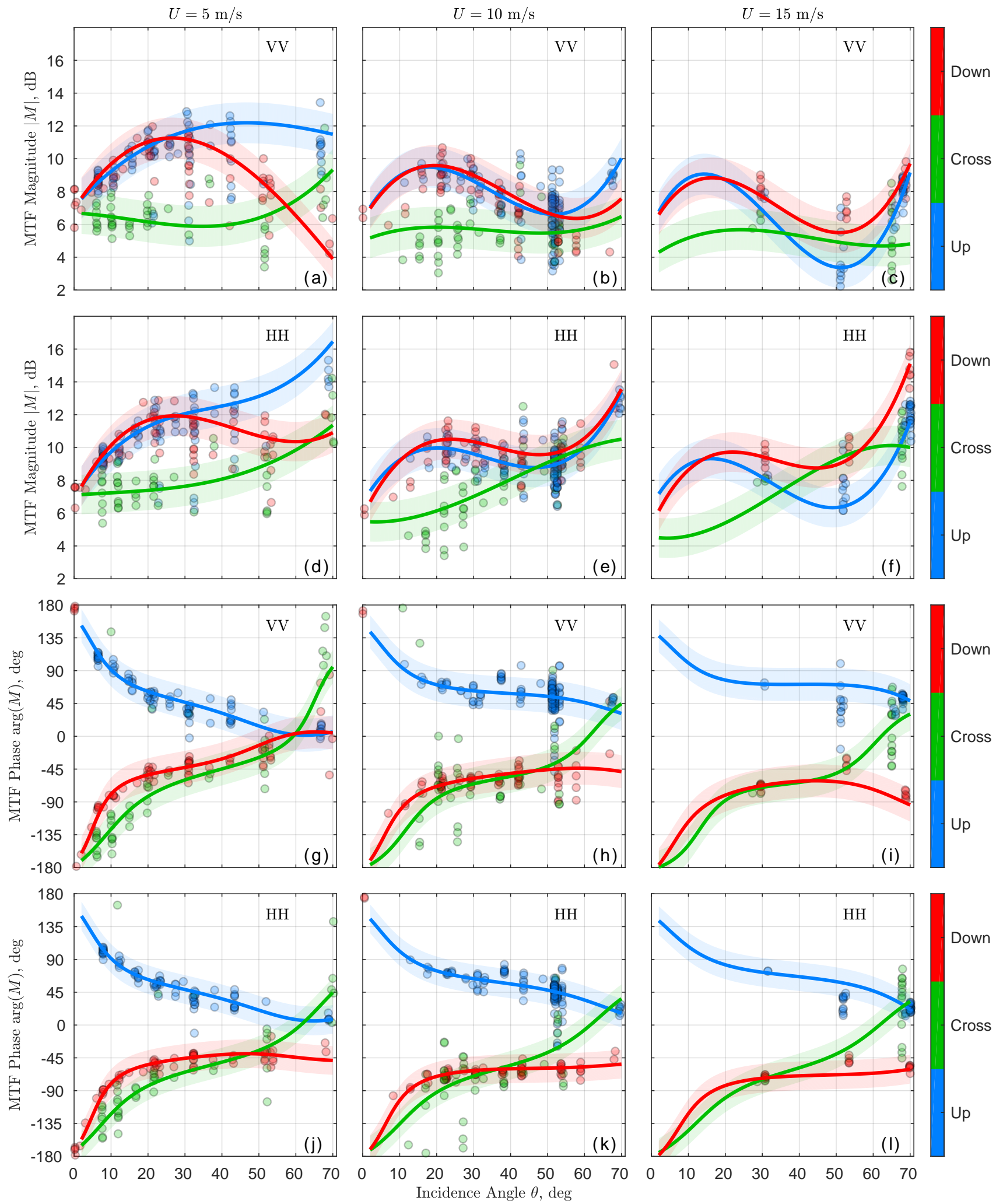

Fig. 9. Measured (circles) and fitted (lines) MTF averaged over resolved wave frequencies, $f_{\mathrm{p}}<f<f_{\text {cut }}$. Measurements are binned in wind speed ( $U \pm 2.5$ $\mathrm{m} / \mathrm{s}$ ) and azimuth $\left(\phi \pm 45^{\circ}\right)$. Confidence intervals (shading) correspond to root-mean-square error of the MTF fit (see Appendix C for details). 

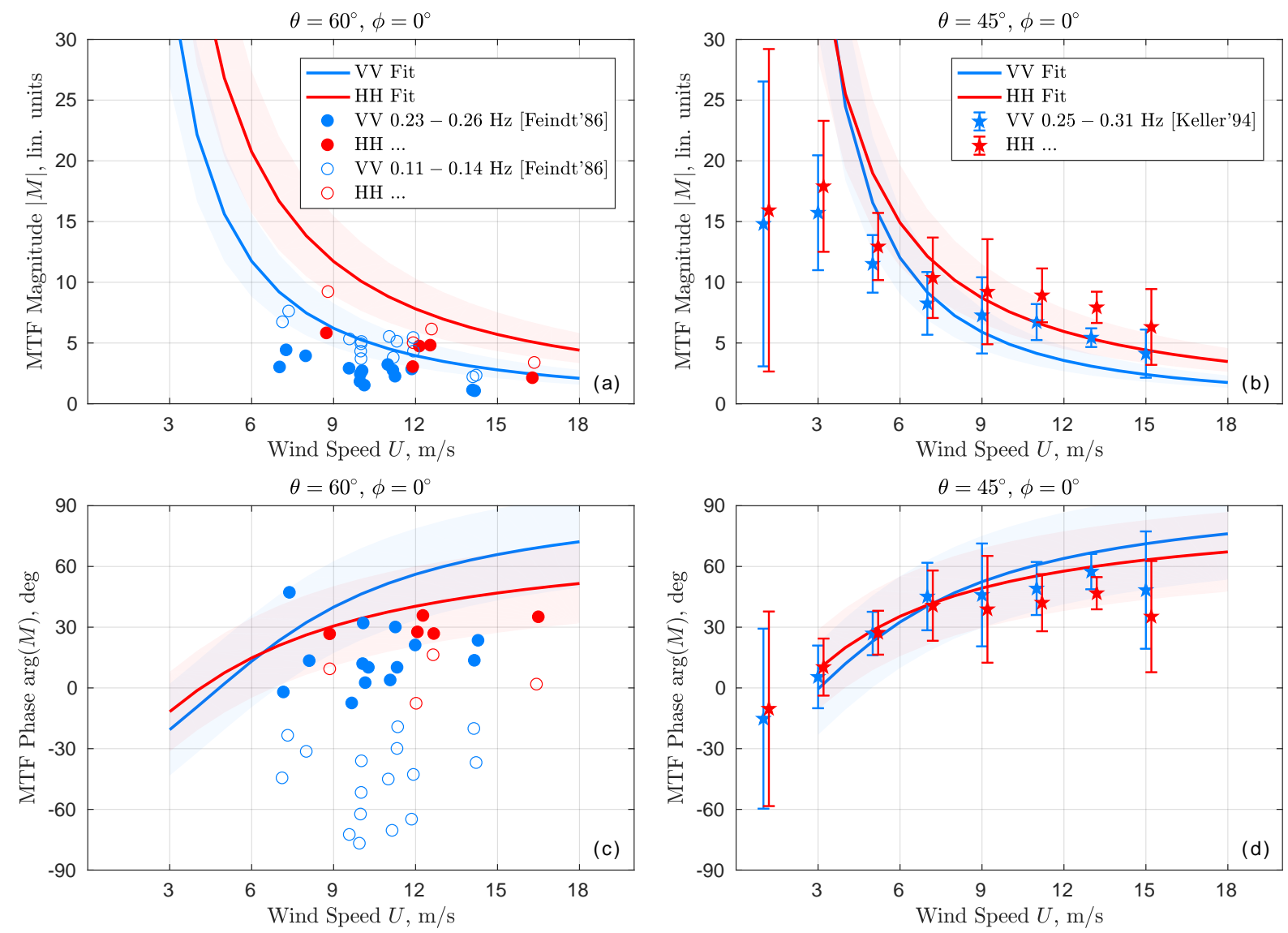

Fig. 10. Upwind MTF fit versus wind speed in comparison with data of (a,c) Feindt et al. [6] (at $\theta=60^{\circ}$ ) and (b,d) Keller et al. [7] (at $\theta=45^{\circ}$ ). Confidence intervals are the same as in Fig. 9.

Feindt et al. [6] data averaged over the swell frequency range agree better with our estimations obtained however for higher frequencies.

MTF phase (Fig. 10c,d) from our observations agrees well with Keller et al. [7] measurements, but is shifted towards wave crests in comparison with Feindt et al. [6]. One of the key points of Feindt et al. [6] is the negative VV MTF phase at low frequencies (swell, shown by empty circles in Fig. 10c). This observation differs from either Keller et al. [7] or our data. Note also that Keller et al. [7] observed negative MTF phases at $\phi=67.5^{\circ}$ (see a middle row in their Fig. 4). This agrees with our azimuthal dependence of MTF phase discussed in Fig. 9.

Although some discrepancies at light winds, possibly resulted from different radar signal-to-noise ratios, our MTF fit generally agrees with Keller et al. [7]. Next, the role of tilt and hydrodynamics MTF components is analyzed in more details.

\section{A. Tilt and Hydrodynamics MTF}

The total MTF can be partitioned into the tilt and hydrodynamics (hydro) components:

$$
M^{\mathrm{pp}}=M_{\mathrm{T}}^{\mathrm{pp}}+M_{\mathrm{H}}^{\mathrm{pp}} .
$$

The tilt-MTF, $M_{\mathrm{T}}^{\mathrm{pp}}$, describes NRCS modulation caused by the LW-induced change of the local incidence angle. To the first order, it can be estimated from the incidence angle dependence of NRCS:

$$
M_{\mathrm{T}}^{\mathrm{pp}}=\frac{1}{\overline{\sigma^{\mathrm{pp}}}} \frac{\partial \sigma^{\mathrm{pp}}}{\partial \theta} \cos \phi .
$$

The tilt-MTF calculated from observed $\overline{\sigma^{\mathrm{pp}}}$ (we used geophysical model function KaDPMod suggested in [18]) is shown in Fig. 11. Its noticeable feature is an inverse behavior at large incidence angles, $M_{\mathrm{T}}^{\mathrm{hh}}>M_{\mathrm{T}}^{\mathrm{vv}}$, that differs from the "expected" Bragg theory behavior. However, this is not surprising because NP backscattering from breaking waves significantly contributes to the NRCS, especially at $\mathrm{HH}$, resulting in a flattening of $\sigma^{\mathrm{hh}}(\theta)$ at large incidence angles (see e.g. [16], their Section 2.2 and their Fig. 2 for more discussion). As discussed in [18], this effect is accounted for by the KaDPMod.

The hydro-MTF, $M_{\mathrm{H}}^{\mathrm{pp}}$, which describes NRCS modulation due to LW-induced modulation of short-scale scatterers, is then obtained by subtracting the $M_{\mathrm{T}}$ from the total MTF:

$$
M_{\mathrm{H}}^{\mathrm{pp}}=M^{\mathrm{pp}}-M_{\mathrm{T}}^{\mathrm{pp}} \text {. }
$$

Observed total, tilt-, and hydro-MTF (both, direct measurements and fits) versus incidence angles and wind speed are shown in Fig. 11. The most striking feature of hydro-MTF is its dependence on polarization that certainly contradicts the 

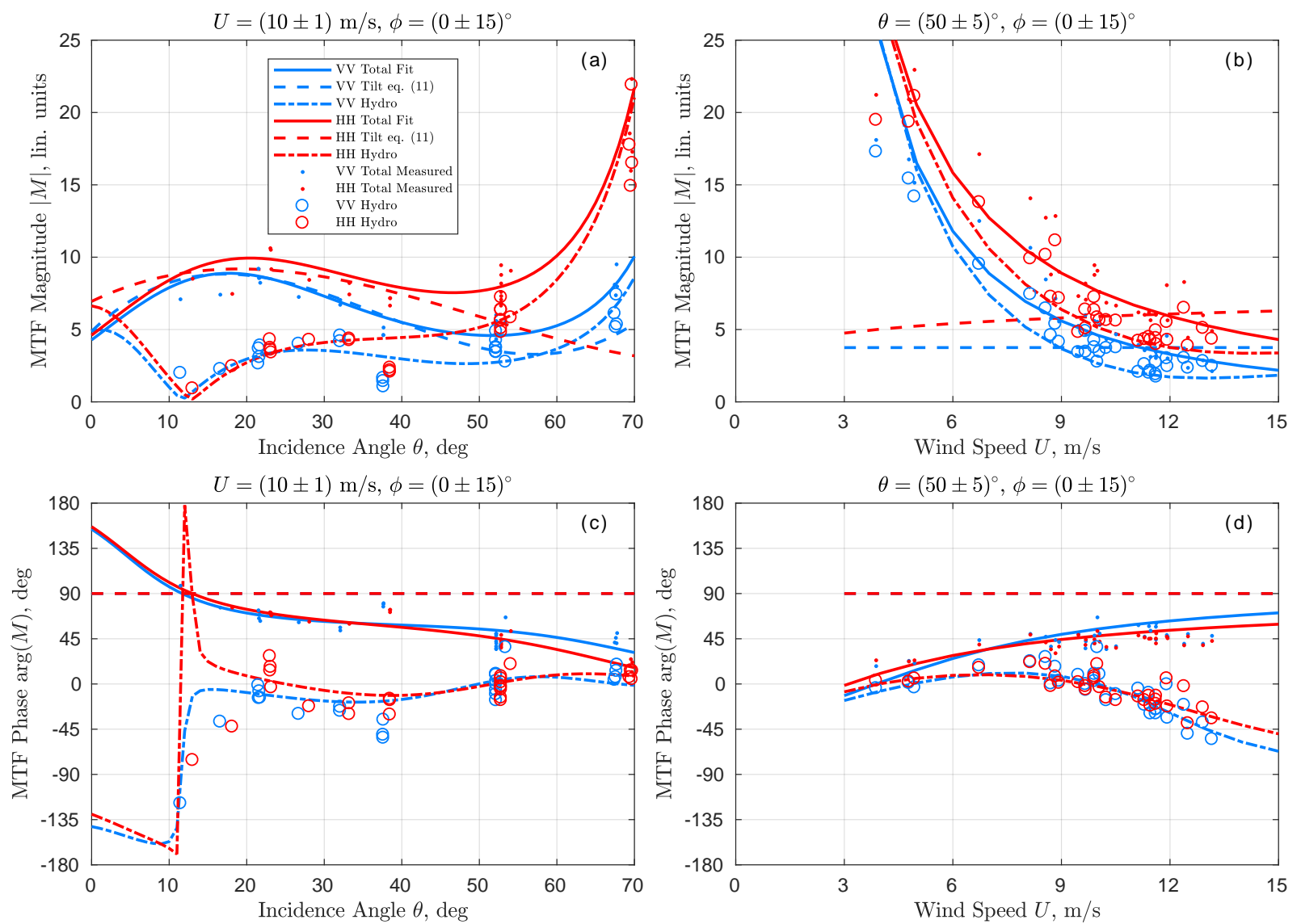

Fig. 11. (a,b) Magnitude and (c,d) phase of total (solid lines - fit, dots - data), tilt (dashed lines - theory), and hydrodynamics (dash-dotted lines - fit, circles - data) MTF for VV (blue) and HH (red) polarization versus (a,c) incidence angle, and (b,d) wind speed.

pure Bragg scattering paradigm. The magnitude of hydro-MTF is systematically higher at $\mathrm{HH}$ polarization (Fig. 11). This is in line with the PR (Fig. 5) which is systematically lower than TSM PR. Lower PR indicates an important role of the NP associated with radar returns from breaking waves (at moderate incidence angles) and specular reflections from "largescale" slopes of regular waves (at low incidence angles). The relative impact of the NP is defined by its partial contribution to the total NRCS, $p^{\mathrm{pp}}=\sigma_{\mathrm{np}} / \sigma^{\mathrm{pp}}$. Since $p^{\mathrm{hh}}>p^{\mathrm{vv}}$ (see, e.g. [18] for more details), the modulation of scattering facets providing NP backscattering has different relative impacts on the hydrodynamics modulation at $\mathrm{VV}$ and $\mathrm{HH}$, and thus results in the polarization dependence of the hydro-MTF. Modulation of short-scale wave breaking by LW is rather strong with MTF magnitudes as large as $\approx 20$ [32], [35]. Such strong wave breaking modulation explains the observed difference between HH and VV hydro-MTF and its increase at large $\theta$ (Fig. 11a).

Another interesting feature of the observed hydro-MTF is its behavior at low incidence angles, $\theta<20^{\circ}$, Fig. 11a,c. First, there is almost no difference between VV and HH hydro-MTF at these $\theta$. This is in line with the dominant NP contribution to the total NRCS at near-nadir $\theta$. Second, the amplitude of hydro-MTF almost zeros and its phase jumps from $0^{\circ}$ to $180^{\circ}$ at around $\theta=13^{\circ}$. This sharp behavior is consistent with so-called "contrast inversion" concept adopted in the sun glitter theory [36], [37]. According to this concept, the hydroMTF is defined by variations of the mean square slope (MSS):

$$
M_{\mathrm{H}}=\left(\tan ^{2} \theta / \zeta^{2}-1\right) M_{\zeta} .
$$

where $\zeta^{2}$ is the large-scale surface wave MSS (in terms of the composite scattering model), and $M_{\zeta}=9 / 4$ is the MSS MTF (see eqs. (12) and (14) in [36]). With $\zeta^{2}=0.053$, this equation describes the observed vanishing of $M_{\mathrm{H}}^{\mathrm{pp}}$ at $\theta=13^{\circ}$.

Observed decrease of $M_{\mathrm{H}}^{\mathrm{pp}}$ amplitude with increasing wind speed (Fig. 11b) is in line with the relaxation theory and associated decrease of the relaxation time (inversely proportional to wind speed squared). But, $M_{\mathrm{H}}^{\mathrm{pp}}$ phase shift from LW crests (at moderate winds) towards backward LW slopes (at higher winds) conflicts the relaxation theory. A possible reason for such discrepancy may be related to wind aerodynamic effects leading to short wave enhancement on the rear (windward) LW slope where the airflow is accelerated. Such shift may also be related to the location and spread of enhanced scattering area during breaking events that lags behind wave crests and thus shifts onto rear slopes of LWs.

\section{B. Partitioning of Hydro-MTF}

Following [19], the NRCS is decomposed as a sum of two components, $\sigma^{\mathrm{pp}}=\sigma_{\mathrm{br}}^{\mathrm{pp}}+\sigma_{\mathrm{np}}$, supported by Bragg scattering, 
$\sigma_{\mathrm{br}}^{\mathrm{pp}}$, and NP scattering, $\sigma_{\mathrm{np}}$. The latter component is related to specular reflection from breaking events at moderate and large $\theta$ and from slopes of regular (non-breaking) waves at low to nadir $\theta$. Synchronous co-polarized measurements enable to separate the two components and investigate them separately [19]. Similarly, the hydro-MTF can also be decomposed into NRCS modulations supported by Bragg and non-Bragg scattering mechanisms:

$$
M_{\mathrm{H}}^{\mathrm{pp}}=\left(1-p^{\mathrm{pp}}\right) M_{\mathrm{br}}^{\mathrm{pp}}+p^{\mathrm{pp}} M_{\mathrm{np}},
$$

where $p^{\mathrm{pp}}=\sigma_{\mathrm{np}} / \sigma^{\mathrm{pp}}$ is the relative NP contribution to the total NRCS, which can be assessed from co-polarized NRCS measurements and theoretical PR for Bragg scattering [18]. Hydro-MTF for Bragg scattering in (14) consists of two terms associated with the pure Bragg wave spectrum MTF and an additional contribution from modulation of the MSS of largescale surface waves (in terms of the two-scale Bragg model, see eq. (19) in [16] for more details),

$$
M_{\mathrm{br}}^{\mathrm{pp}}=M_{0 \mathrm{br}}+\frac{g^{\mathrm{pp}} \zeta^{2}}{1+g^{\mathrm{pp}} \zeta^{2}} M_{\zeta},
$$

where $g^{\mathrm{pp}}$ is the geometrical coefficient (see Appendix B for details). As explained in [16], the effect of MSS modulation on Bragg MTF is weak and can be ignored. Thus, hereinafter, $M_{\mathrm{br}}^{\mathrm{pp}}$ in (15) is solely associated with the pure Bragg MTF, $M_{0 \mathrm{br}}$, which is independent of polarization.

Similarly to the NRCS decomposition [19], the hydroMTF at VV and $\mathrm{HH}$ polarizations, $M_{\mathrm{H}}^{\mathrm{vv}}$ and $M_{\mathrm{H}}^{\mathrm{hh}}$, allows to separate the pure Bragg spectral MTF, $M_{0 \mathrm{br}}$, and MTF for NP scattering, $M_{\mathrm{np}}$ :

$$
\begin{aligned}
M_{0 \mathrm{br}} & =\frac{M_{\mathrm{H}}^{\mathrm{vv}} p^{\mathrm{hh}}-M_{\mathrm{H}}^{\mathrm{hh}} p^{\mathrm{vv}}}{p^{\mathrm{hh}}-p^{\mathrm{vv}}}, \\
M_{\mathrm{np}} & =\frac{M_{\mathrm{H}}^{\mathrm{hh}}\left(1-p^{\mathrm{vv}}\right)-M_{\mathrm{H}}^{\mathrm{vv}}\left(1-p^{\mathrm{hh}}\right)}{p^{\mathrm{hh}}-p^{\mathrm{vv}}} .
\end{aligned}
$$

Estimates of Bragg and NP components of hydro-MTF are accompanied in Fig. 12 by independent measurements of whitecap MTF [32], [38] performed from the same site, but using different optical sensors and techniques. The estimates of MTF components are not shown for $\theta<30^{\circ}$ where specular reflections dominate and partial contributions for $\mathrm{VV}$ and $\mathrm{HH}$ are close. As an alternative, the hydro-MTF based on specular reflection model (13) is shown in Figs. 12a,c for low incidence angles, $\theta<30^{\circ}$, which extends the incidence angle dependence of NP MTF over the entire range of observed $\theta$. This model underestimates MTF magnitude at near nadir angles in comparison with observed values if theoretical MSS MTF is used, $M_{s}=9 / 4$, [36].

Figs. 12a,c suggest that wave breaking MTF and specular reflection MTF (linked to the MSS) are quite consistent and exhibit "smooth" transition between NP modulation by breaking waves (moderate incidence, $\theta>30^{\circ}$ ) and NP modulation by specular reflection from non-breaking waves (at low incidence angles, $\theta<30^{\circ}$ ). At $\theta>13^{\circ}$, the phase of both NP MTFs is close to zero suggesting that either wave breaking or a number of specular points (related to the MSS) is enhanced on the crests of modulating LW. At small incidence angles, $\theta<13^{\circ}$, enhanced MSS results in decreased specular reflectivity, and thus the MTF phase "suddenly" jumps by $180^{\circ}$ towards smoother wave troughs.

The magnitude of wave breaking MTF and magnitude of spectral MTF at the Bragg wavenumber both decrease with wind speed, Fig. 12b. This is explained by the corresponding decrease of the wave relaxation time that becomes much smaller than the period of modulating LW. Wave breaking modulations (inferred from $\mathrm{K}_{\mathrm{a}}$-band radar data) are consistent with whitecap modulations quasi-synchronously measured within the radar footprint [38], though some discrepancies are noticeable at the highest observed wind. Experimental estimates of whitecap coverage from joint optical and wave gauge measurements [32] demonstrate even larger MTF amplitudes. The phase of radar wave breaking MTF indicates an enhancement of breakers over the LW crests. Some tendency indicating a shift towards the rear slope of LW at high winds is also apparent. This tendency is consistent with the observed phase of whitecap MTF (Fig. 12d).

The following qualitative interpretation may be given to explain the observed shift of $M_{\mathrm{np}}$ phase towards rear slopes of the LW. First, it is assumed that wave breaking contributes to the NRCS and is strongly modulated by the LW. Wave breaking crest instability develops over LW crests following short-wave and LW-current interaction. This local area of enhanced instability propagates with LW phase velocity. Yet, once an unstable crest breaks, it generates disturbances and foam embedded in the water in the LW crest area and thus starts moving with the LW orbital velocity. As the LW crest keeps moving forward with its phase speed, these disturbances inevitably shift down the LW rear slope towards its following trough. The resulting enhanced roughness patch responsible for radar backscattering becomes distributed between the LW crest (initial starting point of the instabilities) and the following trough, as observed in Fig. 12d. Note, this interpretation is consistent with unexpectedly low DV corresponding to breaking events (Figs. 4, 5).

Finally, $\mathrm{K}_{\mathrm{a}}$-band Bragg waves (wavelengths from $5 \mathrm{~mm}$ to $10 \mathrm{~mm}$ ) are strongly modulated by LW at low winds (Fig. 11b). Such short ripples are primarily generated as the parasitic, bound capillaries due to the micro-scale breaking of short gravity wave crests, and thus have the phase velocity equal to the phase velocity of the breaking gravity wave [39], [40]. Energy pumping to parasitic capillaries is proportional to the dissipation of short gravity waves (see [16], [40], [41] for more details and references). Modulation of short gravity waves by LW results in modulation of micro-scale breaking and corresponding modulation of parasitic capillaries. Due to the cascade mechanism of capillary wave modulation, the MTF of parasitic capillaries corresponds to the MTF of microscale wave breaking. That is what seems to be revealed in Fig. $11 \mathrm{~b}$ at low winds where the Bragg wave MTF is comparable in magnitude to the NP MTF. At moderate to high winds the "direct generation" of capillary waves by the wind becomes the dominant energy source. Because the relaxation time of capillary waves is much smaller than the period of modulating LW, their modulations tend to disappear at high winds (Fig. 11b). Note, that small amplitude of MTF can lead to unstable 

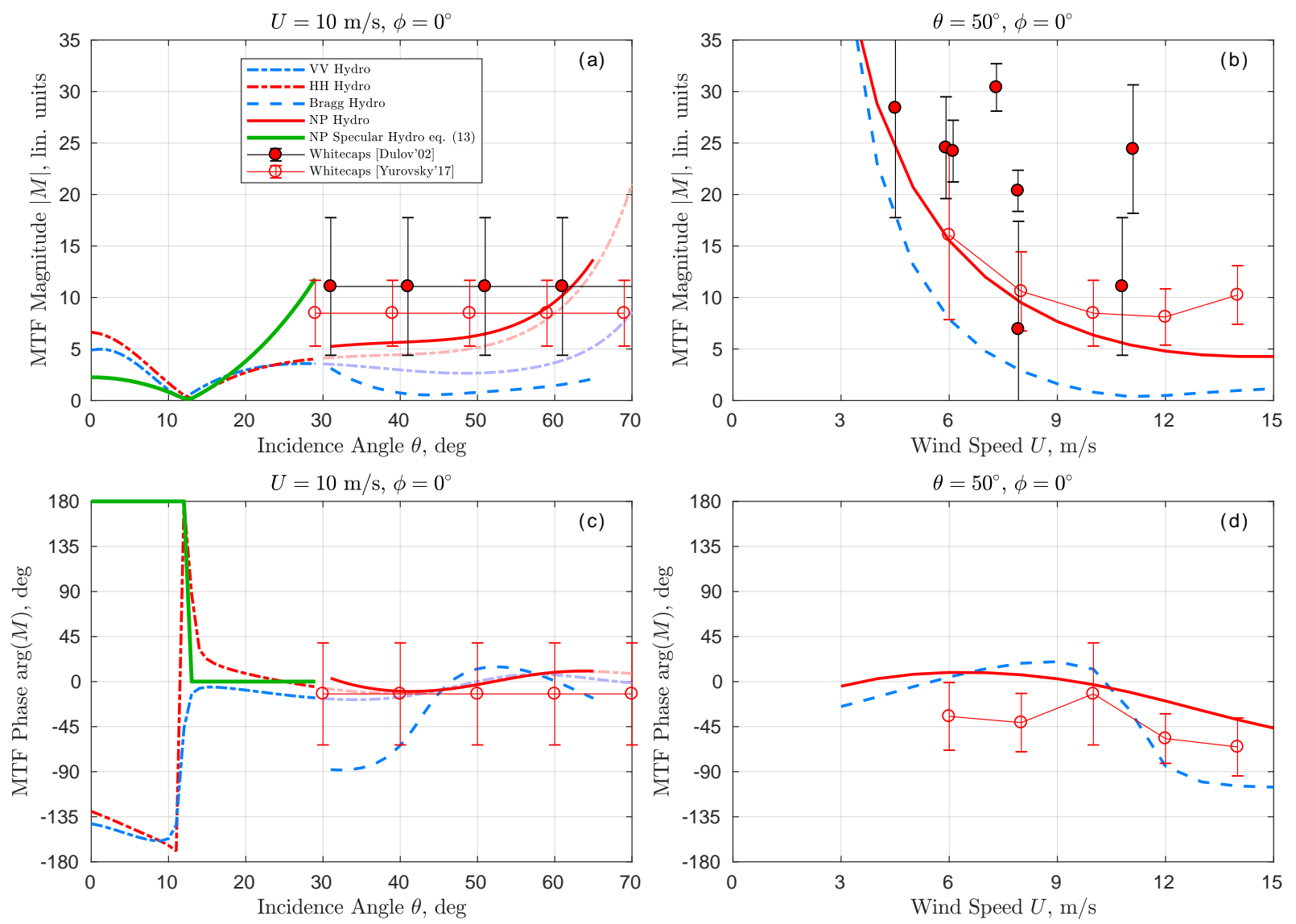

Fig. 12. The same as Fig. 11 for hydro-MTF (dash-dotted lines), Bragg spectral MTF (dashed blue lines), and NP MTF (solid red lines). Green lines are theoretical hydro-MTF (13). Empirical estimates of whitecap MTF from [32] (closed circles) and [38] (open circles).

estimates of its phase. Therefore, MTF phase corresponding to MTF amplitude less than $\approx 2$ can be disregarded. Interestingly, the phase of Bragg MTF is negative at $U>10 \mathrm{~m} / \mathrm{s}$ (maximum of roughness is on the rear, windward wave slope). This negative phase agrees with Feindt et al. [6] who have attributed it to the effect of air flow modulation over the LW. But, as mentioned above, the Bragg MTF phase at $U>10 \mathrm{~m} / \mathrm{s}$ is subject to errors due to the weak Bragg MTF magnitude.

\section{CONCLUSION}

This paper continues the analysis of dual co-polarized (VV and $\mathrm{HH}) \mathrm{K}_{\mathrm{a}}$-band radar backscatter measurements from the sea surface taken from the Black Sea Research platform. While the previous analysis has targeted the time mean properties of $\mathrm{K}_{\mathrm{a}}$-band NRCS [18], the present paper focuses on modulation properties of the NRCS and Doppler Velocity (DV) on spacetime scales corresponding to "long" surface waves (LW) in a frequency range from $0.2 \mathrm{~Hz}$ to $0.8 \mathrm{~Hz}$.

LW-induced variations of the Doppler radar signal, NRCS, and DV, derived from quasi-instantaneous Doppler spectra (averaged over $0.2 \mathrm{sec}$ time intervals), reveal the following remarkable features. First, NRCS and DV modulations are well correlated. Unlike the DV (that is visually quite smooth), the NRCS signal exhibits spiky structure, i.e. large transient enhancements of the NRCS with peaks much larger than the overall background. As expected, HH NRCS modulations are stronger than $\mathrm{VV}$ ones that lead to modulations of the polarization ratio, $\mathrm{PR}=\mathrm{VV} / \mathrm{HH}$. This suggests that important source of NRCS modulation is the modulation of scattering facets providing non-polarized (NP) radar return. These facets likely correspond to wave breaking events at moderate and large $\theta$ or to specular surface slopes at low to nadir $\theta$.

The statistical distribution of LW-induced variations of the NRCS and DV are different. The probability density function (PDF) of the NRCS (in linear units) is strongly skewed in a wide range of incidence angles that confirms the presence of spike-like events associated with wave breaking. In contrast, the skewness of DV PDF is much smaller at moderate and low incidence angles. This suggests that DV of NRCS spikes is "low", i.e. it is not locked to the phase velocity of breaking waves. As a consequence, LW elevation spectra derived from DV measurements are very close to LW elevation spectra measured by the wave gauge. Unlike, at large incidence angles $\left(\theta=70^{\circ}\right)$ and $\mathrm{HH}$ polarization, the DV PDF is strongly skewed and DV-derived LW elevation spectra deviate significantly from wave gauge elevation spectra. This indicates that breakers are detected by a radar as "fast scatterers" at large incidence angles.

In order to reconcile DV measurements at moderate and large incidence angles, the following paradigm is suggested. 
First, it is assumed that a breaker advances with the phase velocity and leaves behind an expanding area of enhanced surface roughness "embedded in the water". Forward faces of active breakers are exposed at large incidence angles and in the upwind direction. At these observation conditions, NRCS peaks are formed by the fast scatters that leads to strong asymmetry of Doppler spectra and deviation between DV-derived and wave gauge elevation spectra. In contrast, at moderate incidence angles, NRCS spikes correspond to the slow facets (embedded into the water) on the tops of breakers, which leads neither to significant asymmetry of Doppler spectra nor apparent deviation between DV-derived and wave gauge spectra.

After splitting the MTF into tilt- and hydro-components, the hydro-MTF is found to be polarization dependent with higher $\mathrm{HH}$ amplitudes. This certainly contradicts the standard Bragg scattering theory and points to the non-negligible contribution of NP scattering from wave breaking. Depending on wind speed, the NP modulations are significant with MTF amplitude varying from 5 to about 30. At low to moderate winds, breakers locate on the crests of modulating LW. Above 10 $\mathrm{m} / \mathrm{s}$, the maximum of breaker modulation shifts towards the rear LW slopes.

At low incidence angles $\left(\theta<30^{\circ}\right)$, NP scattering is dominated by specular reflections from slopes of regular (nonbreaking) waves. The observed hydro-MTF at such $\theta$ exhibits features anticipated from the quasi-specular reflection theory. In particular, its amplitude zeros in the vicinity of the incidence angle corresponding to mean square slope of the large-scale surface waves. This is accompanied by an abrupt change of the MTF phase by $180^{\circ}$. Combination of observed wave breaking $\operatorname{MTF}\left(\theta>30^{\circ}\right)$ and theoretical specular point $\operatorname{MTF}\left(\theta<30^{\circ}\right)$ provides a proxy for a consistent description of the NP hydroMTF in the full range of observed incidence angles.

The spectral MTF of the resonant Bragg scattering is essential only at low winds where its magnitude is comparable to the magnitude of the wave breaking MTF. Because $\mathrm{K}_{\mathrm{a}}$ band Bragg waves are parasitic capillaries at low winds, their modulations are related to modulations of micro-scale breaking of short gravity waves. At winds above $8 \mathrm{~m} / \mathrm{s}$, the direct generation by wind becomes increasingly important for $\mathrm{K}_{\mathrm{a}}$-band Bragg millimeter scale waves, and thus their modulation is significantly suppressed by wind forcing and weakly contributes to the hydro-MTF.

These observations provide deeper insights into the physics of surface wave modulations and millimeter wavelength radar backscattering from the ocean surface. This is needed for development of new space-borne $\mathrm{K}_{\mathrm{a}}$-band instruments and better retrieval of the sea surface characteristics via a synergy of microwave and optical remote sensing techniques (e.g. [42], [43]). Proposed parameterization of the $\mathrm{K}_{\mathrm{a}}$-band radar MTF can further be used to assess the wave-induced contribution to the mean DV. This is essential for better separation of Doppler shift components due to ocean currents and waveinduced motions.

\section{APPENDIX A \\ CORRECTION FOR THE RADAR PATTERN}

Spatial filtering by radar pattern (RP) footprint is represented as the wave number filter response function [44]:

$$
\gamma(\mathbf{k})=\frac{|\operatorname{FFT} 2(\gamma(\mathbf{x}))|^{2}}{<\gamma(\mathbf{x})>},
$$

where $\mathbf{k}$ is the wave number vector, $\langle\ldots\rangle$ denotes spatial averaging, $\gamma(\mathbf{x})$ is the two-way RP projected on the sea surface plane and accounting for the $R^{-4}$ distance fading and the incidence angle dependence of NRCS:

$$
\gamma(\mathbf{x})=\frac{\Gamma(\mathbf{x}) R(\mathbf{x})^{-4} \sigma(\mathbf{x})}{R\left(\mathbf{x}_{0}\right)^{-4} \sigma\left(\mathbf{x}_{0}\right)},
$$

where $\mathbf{x}$ is the local point on the sea surface, $\mathbf{x}_{0}$ is the intersection point of the radar axis and sea surface, $R$ is the distance from the radar to the local point on the sea surface, $\sigma$ is the NRCS at the local point (evaluated from NRCS model [18]), $\Gamma$ is the two-way RP known from calibration (see Appendix in [18] for details) and projected on the sea surface. Examples of RP projection, $\gamma(\mathbf{x})$, and its wave number response function, $\gamma(\mathbf{k})$, are given in Fig. 13.

Wave number response function, $\gamma(\mathbf{k})$, is then projected onto frequency-azimuth space using the deep water dispersion relationship, $(2 \pi f)^{2}=g k$, yielding frequency-azimuth response function, $\gamma(f, \phi)$, that is used for the correction of radar spectra at given $\theta, \phi, U$ :

$$
S_{p q}^{\prime}(f)=S_{p q}(f) \gamma(f)^{-1},
$$

where $p, q$ are either DV, $v$, or NRCS, $\sigma$.

The above correction is important only for the radar-derived elevation spectra, $S_{\xi \xi}$, eq. (8), and the DV response function, $\mu$, eq. (6). For the traditional MTF, $M$, eq. (4), the corrections included in the nominator and denominator cancel each other.

Specific antenna design results in different VV and $\mathrm{HH}$ response functions (Fig. 13,c,d). In particular, $\mathrm{HH}$ channel passes higher/lower frequencies than VV channel in the radarlook/cross-look direction, respectively. The cutoff frequency of RP frequency response function (Fig. 14a) decreases from 0.5 $\mathrm{Hz}$ at near-nadir $\theta$ to $0.25 \mathrm{~Hz}$ at $\theta=70^{\circ}$. This study analysis is limited to frequencies lower than the cutoff frequency, $f_{\text {cut }}$ corresponding to 0.25 of the maximum RP response magnitude. At $f<f_{\text {cut }}$, the correction (20) effectively accounts for the effect of RP spatial filtering.

Finite RP angular width along with strong incidence angle dependence of the sea surface NRCS, result in shifting of the footprint centroid, $\left.\mathbf{x}_{\text {eff }}=\int \mathbf{x} \gamma(\mathbf{x}) \mathrm{d} \mathbf{x}\right) / \int \gamma(\mathbf{x}) \mathrm{d} \mathbf{x}$, and corresponding decrease of the effective incidence angle, $\theta_{\text {eff }}=$ $\arctan \left(\left|\mathbf{x}_{\mathrm{eff}}\right| / H\right)$, where $H$ is the radar height. Deviation of the nominal incidence angle (corresponding to geometrical radar axis) from the effective one, $\theta-\theta_{\text {eff }}$, is always positive and peaks at $\theta \approx 20^{\circ}$, where $\sigma(\theta)$ has the largest gradient (Fig. $14 \mathrm{~b})$. At high incidence angles, the deviation increases because of rapid distance fading, $R^{-4}$. This deviation is stronger for VV polarization because the angular width of VV RP is larger than that of HH RP in the range direction. Typical deviation from the nominal $\theta$ is below $3.5^{\circ}$ and $2^{\circ}$ for $\mathrm{VV}$ and $\mathrm{HH}$ 

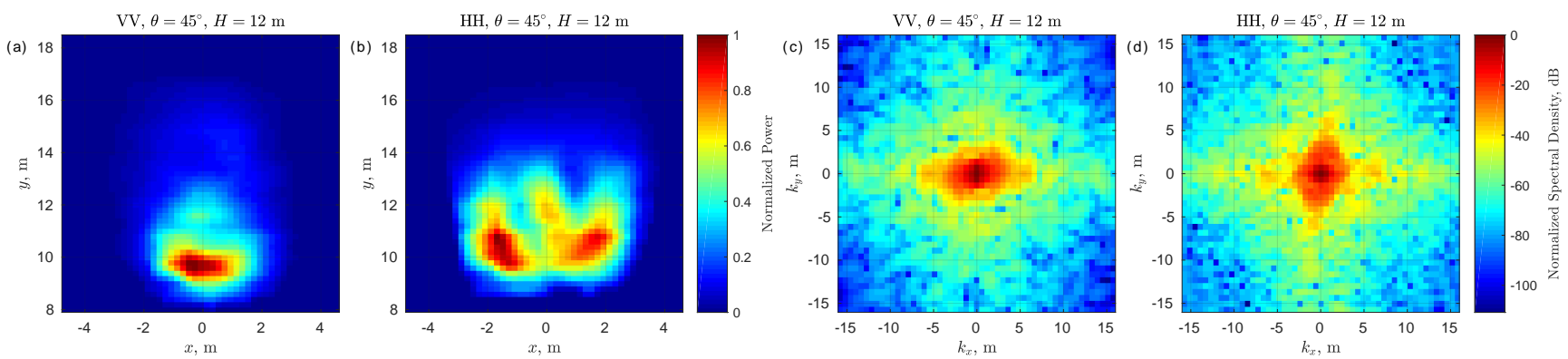

Fig. 13. (a,b) Example of radar pattern projection on the sea surface and $(c, d)$ its response in the wave number space for $(a, c)$ VV and (b,d) HH polarizations. Incidence angle is $45^{\circ}$, upwind azimuth, $10 \mathrm{~m} / \mathrm{s}$ wind speed, radar height is $12 \mathrm{~m}$.
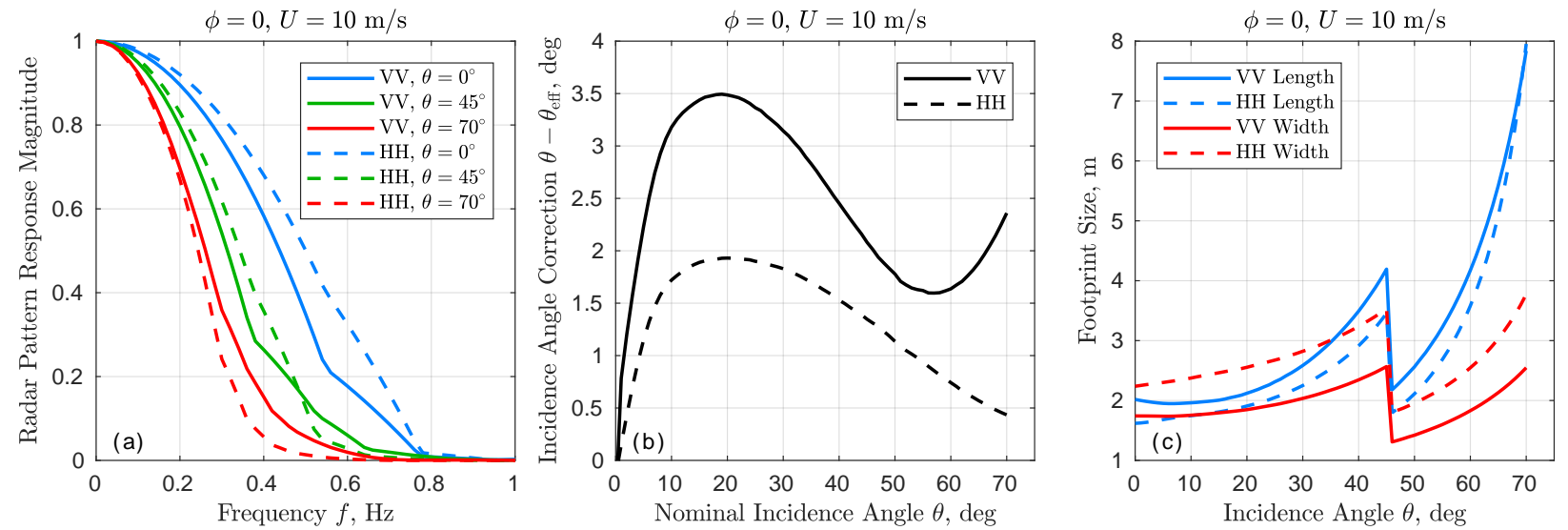

Fig. 14. (a) Radar pattern response versus wave frequency, (b) incidence angle correction, $\theta-\theta_{\text {eff }}$, versus nominal incidence angle $\theta$, (c) $-3 \mathrm{~dB}$ ground radar footprint size (cross-look width and in-look length) versus incidence angle (the jump at $\theta=45^{\circ}$ corresponds to the change in radar height from $12 \mathrm{~m}$ to $6 \mathrm{~m})$.

polarization, respectively, at $U=10 \mathrm{~m} / \mathrm{s}$. The above correction is applied to all analyzed data.

Ground size of RP footprint in the range (length) and the cross-look (width) directions translates into the corresponding RP frequency response (Fig. 14c). The jump in RP footprint size is caused by change in radar height at $\theta=45^{\circ}$.

\section{APPENDIX B \\ Two-Scale Model Evaluation}

The Bragg theory prediction of the polarization ratio (PR) is based on the classical Two-Scale Model (TSM, [45], [46]) that involves a separation of wave scales onto short resonant Bragg waves with wave number, $k_{\mathrm{br}}=2 k_{\mathrm{r}} \sin \theta$, and longer modulating waves, $k<k_{\mathrm{br}} / 4$. TSM PR estimate follows [41] and is based on the following set of equations:

$$
\begin{aligned}
\mathrm{PR}_{\mathrm{br}} & =\frac{\left|G_{\mathrm{vv}}\right|^{2}\left(1+g_{\mathrm{vv}} \zeta_{\mathrm{i}}^{2}\right)}{\left|G_{\mathrm{hh}}\right|^{2}\left(1+g_{\mathrm{hh}} \zeta_{\mathrm{i}}^{2}\right)}, \\
g_{\mathrm{vv}} & =\frac{1}{2\left|G_{\mathrm{vv}}\right|^{2}} \frac{\partial^{2}\left|G_{\mathrm{vv}}\right|^{2}}{\partial \theta^{2}}, \\
g_{\mathrm{hh}} & =\frac{1}{2\left|G_{\mathrm{hh}}\right|^{2}} \frac{\partial^{2}\left|G_{\mathrm{hh}}\right|^{2}}{\partial \theta^{2}}+\frac{2}{\sin ^{2} \theta} \frac{\left|G_{\mathrm{vv}}\right| G_{\mathrm{hh}} \mid}{\zeta_{\mathrm{c}}^{2}} \\
\zeta_{\mathrm{iv}}^{2} & =\frac{\left[\epsilon+(\epsilon-1) \sin ^{2} \theta\right](\epsilon-1) \cos ^{2} \theta}{\left[\epsilon \cos \theta+\left(\epsilon-\sin ^{2} \theta\right)^{1 / 2}\right]^{2}}, \\
G_{\mathrm{hh}} & =\frac{(\epsilon-1) \cos ^{2} \theta}{\left[\cos \theta+\left(\epsilon-\sin ^{2} \theta\right)^{1 / 2}\right]^{2}},
\end{aligned}
$$

where $\epsilon=17-i 28$ is the complex dielectric constant of sea water in the $\mathrm{K}_{\mathrm{a}}$-band for $20^{\circ}$ temperature and $18 \mathrm{~g} / \mathrm{kg}$ salinity [47], $\zeta_{\mathrm{i}}^{2}$ and $\zeta_{\mathrm{c}}^{2}$ is the MSS of modulating waves in the incidence plane and in the plane normal to the incidence plane, respectively.

\section{APPENDIX C \\ FITTING OF THE MTF}

MTF fitting function is based on empirical polynomials of incidence angle, $\theta$, azimuth, $\phi$, and wind speed, $U$. The fitting 

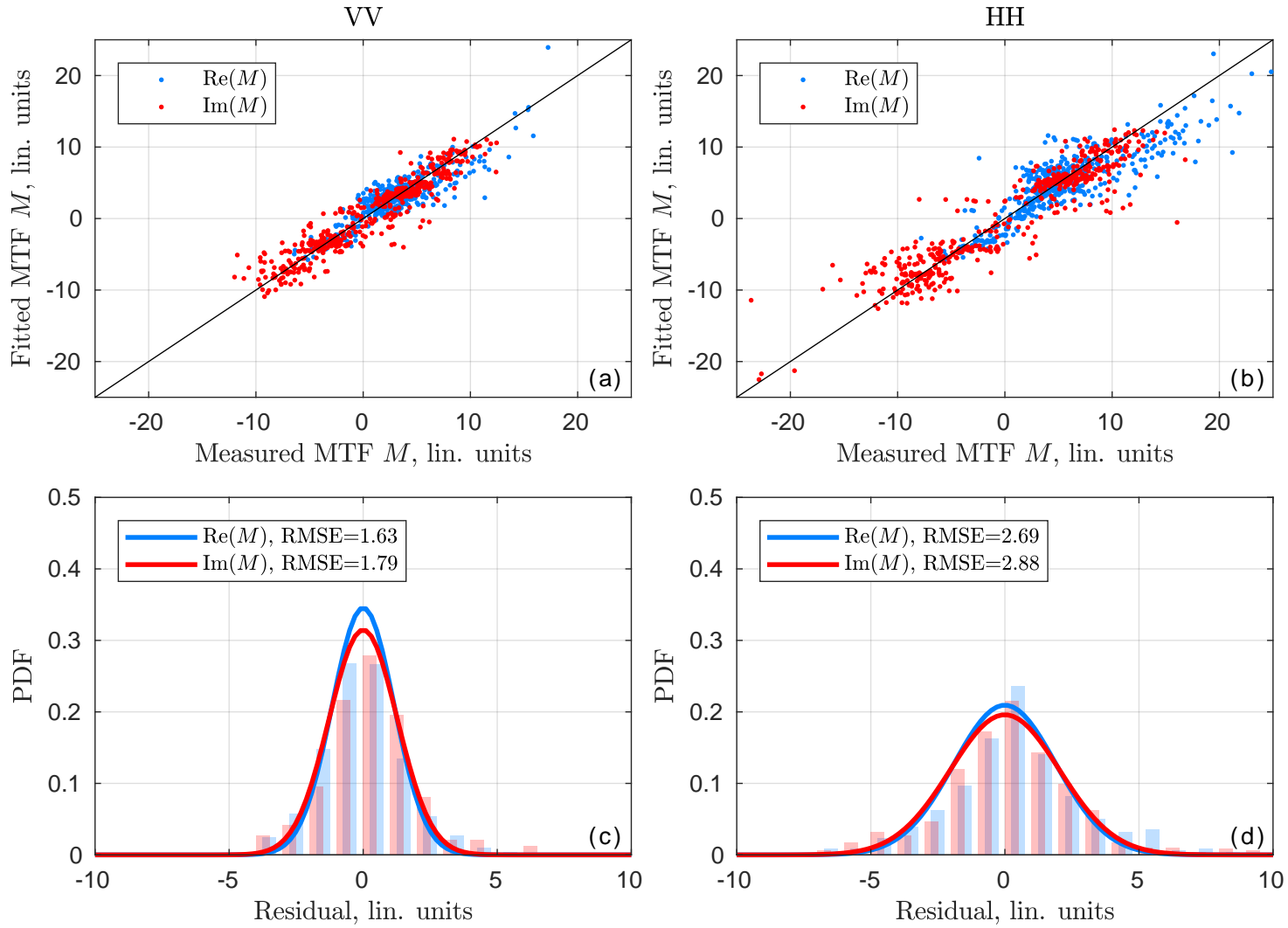

Fig. 15. (a,b) Scatter plots for fitted versus measured MTF, (c,d) observed (bars) and gauss-fitted (lines) residual distributions. Left column (a,c) - VV polarization, right column $(\mathrm{b}, \mathrm{d})-\mathrm{HH}$ polarization. Blue color is real part of MTF, red color is imaginary part of MTF.

improves if magnitude and phase are fitted independently. To account for the wide range of MTF magnitude, it is fitted in logarithmic units (dB). Magnitude, $\log (|M|)$, and phase, $M /|M|$, are fitted as:

$$
\left[\begin{array}{c}
\log (|M|) \\
M /|M|
\end{array}\right]=\sum_{i=0}^{n} \sum_{j=0}^{2} \sum_{k=0}^{1}\left[\begin{array}{l}
B_{i j k} \\
C_{i j k}
\end{array}\right] \theta^{i} \cos (j \phi)(\log U)^{k},
$$

where $n=3$ is the polynomial degree selected empirically. Resulting fit represents MTF magnitude in dB units (Fig. 9). For further analysis, it is converted back to linear units (Fig. $11)$.

The distribution of fitting residuals (Fig.15) is quasiGaussian as suggested by $\chi^{2}$-tests (Table I). But, RMSE of $\mathrm{HH}$ fit is larger than that of VV fit. This may be a consequence of unresolved variability present in HH signal. In particular, wave breaking leads to more spiky HH backscattering, which is less suitable for the linear MTF analysis. RMSE for MTF magnitude and phase as well as for real and imaginary MTF components (Table I) are used to plot confidence intervals in Fig. 9. Coefficients $B_{i j k}$ and $C_{i j k}$ from eq. (26) are given in Table II.

\section{ACKNOWLEDGMENT}

The core support of the work was provided by Russian Science Foundation grant No. 15-17-20020. Field experiments
TABLE I

Root-MeAN-Square ERrors AND ChI-SQUare TeStS of MTF Fit

\begin{tabular}{|l|c|c|c|c|}
\hline Parameter & VV RMSE & HH RMSE & VV $\chi^{2}$ & HH $\chi^{2}$ \\
\hline$|M|, \mathrm{dB}$ & 1.25 & 1.21 & 3.48 & 3.06 \\
$|M|$, lin. units & 1.69 & 2.81 & 11.81 & 10.81 \\
$\arg (M)$, deg & 22.69 & 19.69 & 0.62 & 1.49 \\
$\operatorname{Re}(M)$, lin. units & 1.63 & 2.69 & 5.14 & 5.78 \\
$\operatorname{Im}(M)$, lin. units & 1.79 & 2.88 & 8.98 & 6.38 \\
\hline
\end{tabular}

Critical $\chi^{2}$ for $5 \%$ significance level and 7 degrees of freedom is 14.07 .

in 2014-2015 were supported by FASO of Russia under the State Assignment (No. 0827-2014-0010). At the final stage, this work was partly supported by NASA NNX15AG40G and the Ministry of Science and Education via Goszadanie 5.2928.2017/PP. The authors would like to thank E. Rodriguez, B. Plant, W. Alpers, and anonymous referees who made very helpful comments and suggestions leading to improvements of this paper.

\section{REFERENCES}

[1] B. Chapron, F. Collard, and F. Ardhuin, "Direct measurements of ocean surface velocity from space: Interpretation and validation," J. Geophys. Res. Oceans, vol. 110, C07008, 2005, doi:10.1029/2004JC002809.

[2] A. A. Mouche, F. Collard, B. Chapron, K. F. Dagestad, G. Guitton, J. A. Johannessen, V. Kerbaol, and M. W. Hansen, "On the Use of Doppler Shift for Sea Surface Wind Retrieval From SAR," IEEE Trans. Geosci. Remote Sens., vol. 50, no. 7, pp. 2901-2909, July 2012. 
TABLE II

COEFFICIENTS $B_{i j k}$ AND $C_{i j k}$

\begin{tabular}{|c|c|c|c|c|c|c|c|c|}
\hline \multicolumn{3}{|c|}{ Index } & \multicolumn{3}{|c|}{ VV polarization } & \multicolumn{3}{|c|}{$\mathrm{HH}$ polarization } \\
\hline$i$ & $j$ & $k$ & $B_{i j k}$ & $\operatorname{Re}\left(C_{i j k}\right)$ & $\operatorname{Im}\left(C_{i j k}\right)$ & $B_{i j k}$ & $\operatorname{Re}\left(C_{i j k}\right)$ & $\operatorname{Im}\left(C_{i j k}\right)$ \\
\hline 0 & 0 & 0 & $+2.224 \cdot 10^{0}$ & $-1.184 \cdot 10^{0}$ & $-2.027 \cdot 10^{-1}$ & $+2.214 \cdot 10^{0}$ & $-1.122 \cdot 10^{0}$ & $-2.347 \cdot 10^{-1}$ \\
\hline 1 & 0 & 0 & $-2.069 \cdot 10^{-2}$ & $+1.339 \cdot 10^{-1}$ & $-4.231 \cdot 10^{-2}$ & $+4.959 \cdot 10^{-2}$ & $+1.588 \cdot 10^{-1}$ & $-4.202 \cdot 10^{-2}$ \\
\hline 2 & 0 & 0 & $+1.972 \cdot 10^{-3}$ & $-1.967 \cdot 10^{-3}$ & $+1.006 \cdot 10^{-3}$ & $-1.057 \cdot 10^{-3}$ & $-3.469 \cdot 10^{-3}$ & $+1.198 \cdot 10^{-3}$ \\
\hline 3 & 0 & 0 & $-2.336 \cdot 10^{-5}$ & $+6.323 \cdot 10^{-6}$ & $-3.506 \cdot 10^{-6}$ & $+7.777 \cdot 10^{-6}$ & $+2.335 \cdot 10^{-5}$ & $-9.005 \cdot 10^{-6}$ \\
\hline 0 & 1 & 0 & $+2.880 \cdot 10^{-2}$ & $-1.267 \cdot 10^{-1}$ & $+1.135 \cdot 10^{-1}$ & $-1.491 \cdot 10^{-1}$ & $-1.542 \cdot 10^{-1}$ & $+3.113 \cdot 10^{-1}$ \\
\hline 1 & 1 & 0 & $-4.146 \cdot 10^{-2}$ & $-3.090 \cdot 10^{-2}$ & $+1.416 \cdot 10^{-1}$ & $-1.183 \cdot 10^{-2}$ & $-1.595 \cdot 10^{-2}$ & $+1.313 \cdot 10^{-1}$ \\
\hline 2 & 1 & 0 & $+2.106 \cdot 10^{-3}$ & $+1.790 \cdot 10^{-3}$ & $-5.720 \cdot 10^{-3}$ & $+1.003 \cdot 10^{-3}$ & $+1.088 \cdot 10^{-3}$ & $-5.934 \cdot 10^{-3}$ \\
\hline 3 & 1 & 0 & $-1.515 \cdot 10^{-5}$ & $-2.170 \cdot 10^{-5}$ & $+4.877 \cdot 10^{-5}$ & $-5.136 \cdot 10^{-6}$ & $-1.345 \cdot 10^{-5}$ & $+6.030 \cdot 10^{-5}$ \\
\hline 0 & 2 & 0 & $-2.262 \cdot 10^{-1}$ & $-8.339 \cdot 10^{-2}$ & $-4.235 \cdot 10^{-2}$ & $-2.741 \cdot 10^{-1}$ & $-7.071 \cdot 10^{-2}$ & $+5.578 \cdot 10^{-2}$ \\
\hline 1 & 2 & 0 & $+3.944 \cdot 10^{-2}$ & $+9.010 \cdot 10^{-2}$ & $+8.950 \cdot 10^{-2}$ & $+2.573 \cdot 10^{-2}$ & $+6.652 \cdot 10^{-2}$ & $+6.089 \cdot 10^{-2}$ \\
\hline 2 & 2 & 0 & $+1.220 \cdot 10^{-3}$ & $-3.965 \cdot 10^{-3}$ & $-2.931 \cdot 10^{-3}$ & $+9.389 \cdot 10^{-4}$ & $-2.622 \cdot 10^{-3}$ & $-1.497 \cdot 10^{-3}$ \\
\hline 3 & 2 & 0 & $-2.845 \cdot 10^{-5}$ & $+4.488 \cdot 10^{-5}$ & $+2.351 \cdot 10^{-5}$ & $-1.758 \cdot 10^{-5}$ & $+2.668 \cdot 10^{-5}$ & $+6.191 \cdot 10^{-6}$ \\
\hline 0 & 0 & 1 & $-4.021 \cdot 10^{-1}$ & $+6.347 \cdot 10^{-2}$ & $+1.426 \cdot 10^{-1}$ & $-3.753 \cdot 10^{-1}$ & $+5.339 \cdot 10^{-2}$ & $+1.345 \cdot 10^{-1}$ \\
\hline 1 & 0 & 1 & $+3.357 \cdot 10^{-2}$ & $-1.800 \cdot 10^{-2}$ & $+3.808 \cdot 10^{-3}$ & $-1.226 \cdot 10^{-3}$ & $-3.355 \cdot 10^{-2}$ & $+3.799 \cdot 10^{-3}$ \\
\hline 2 & 0 & 1 & $-1.741 \cdot 10^{-3}$ & $+8.967 \cdot 10^{-5}$ & $-1.564 \cdot 10^{-4}$ & $-8.898 \cdot 10^{-5}$ & $+8.825 \cdot 10^{-4}$ & $-2.194 \cdot 10^{-4}$ \\
\hline 3 & 0 & 1 & $+1.835 \cdot 10^{-5}$ & $+2.085 \cdot 10^{-6}$ & $+6.570 \cdot 10^{-7}$ & $+2.093 \cdot 10^{-6}$ & $-6.237 \cdot 10^{-6}$ & $+2.557 \cdot 10^{-6}$ \\
\hline 0 & 1 & 1 & $-9.136 \cdot 10^{-3}$ & $+5.785 \cdot 10^{-2}$ & $+3.167 \cdot 10^{-2}$ & $+1.064 \cdot 10^{-1}$ & $+8.523 \cdot 10^{-2}$ & $-6.176 \cdot 10^{-2}$ \\
\hline 1 & 1 & 1 & $+1.886 \cdot 10^{-2}$ & $+1.437 \cdot 10^{-2}$ & $-3.362 \cdot 10^{-2}$ & $+6.039 \cdot 10^{-4}$ & $+3.372 \cdot 10^{-3}$ & $-2.916 \cdot 10^{-2}$ \\
\hline 2 & 1 & 1 & $-1.015 \cdot 10^{-3}$ & $-8.507 \cdot 10^{-4}$ & $+1.778 \cdot 10^{-3}$ & $-3.646 \cdot 10^{-4}$ & $-3.287 \cdot 10^{-4}$ & $+1.894 \cdot 10^{-3}$ \\
\hline 3 & 1 & 1 & $+8.185 \cdot 10^{-6}$ & $+1.048 \cdot 10^{-5}$ & $-1.632 \cdot 10^{-5}$ & $+1.992 \cdot 10^{-6}$ & $+4.641 \cdot 10^{-6}$ & $-2.154 \cdot 10^{-5}$ \\
\hline 0 & 2 & 1 & $+1.626 \cdot 10^{-1}$ & $+1.145 \cdot 10^{-1}$ & $+6.235 \cdot 10^{-2}$ & $+1.550 \cdot 10^{-1}$ & $+8.275 \cdot 10^{-2}$ & $+2.749 \cdot 10^{-2}$ \\
\hline 1 & 2 & 1 & $-2.149 \cdot 10^{-3}$ & $-3.875 \cdot 10^{-2}$ & $-3.247 \cdot 10^{-2}$ & $+1.127 \cdot 10^{-2}$ & $-2.423 \cdot 10^{-2}$ & $-1.748 \cdot 10^{-2}$ \\
\hline 2 & 2 & 1 & $-1.076 \cdot 10^{-3}$ & $+1.640 \cdot 10^{-3}$ & $+1.260 \cdot 10^{-3}$ & $-1.276 \cdot 10^{-3}$ & $+8.987 \cdot 10^{-4}$ & $+5.322 \cdot 10^{-4}$ \\
\hline 3 & 2 & 1 & $+1.725 \cdot 10^{-5}$ & $-1.856 \cdot 10^{-5}$ & $-1.184 \cdot 10^{-5}$ & $+1.578 \cdot 10^{-5}$ & $-9.139 \cdot 10^{-6}$ & $-3.502 \cdot 10^{-6}$ \\
\hline
\end{tabular}

[3] A. Martin and C. Gommenginger, "Towards wide-swath high-resolution mapping of total ocean surface current vectors from space: Airborne proof-of-concept and validation," Remote Sens. Env., vol. 197, pp. 58-71, 2017.

[4] E. Rodriguez, D. Perkovic-Martin, C. Baldi, K. Cooper, N. Majurec, M. Neumann, F. Nicaise, and G. Farquharson, "Ka-band doppler scatterometer for measurements of ocean surface vector winds and currents," in Proc. Earth Sci. Tech. Forum ESTF2014, Leesburg, Virginia, USA, October 2014.

[5] F. Ardhuin, Y. Aksenov, A. Benetazzo, L. Bertino, P. Brandt, E. Caubet, B. Chapron, F. Collard, S. Cravatte, F. Dias, G. Dibarboure, L. Gaultier, J. Johannessen, A. Korosov, G. Manucharyan, D. Menemenlis, M. Menendez, G. Monnier, A. Mouche, F. Nouguier, G. Nurser, P. Rampal, A. Reniers, E. Rodriguez, J. Stopa, C. Tison, M. Tissier, C. Ubelmann, E. van Sebille, J. Vialard, and J. Xie, "Measuring currents, ice drift, and waves from space: the Sea Surface KInematics Multiscale monitoring (SKIM) concept," Ocean Sci. Discus., vol. 2017, pp. 1-26, 2017.

[6] F. Feindt, J. Schroeter, and W. Alpers, "Measurement of the ocean wave-radar modulation transfer function at $35 \mathrm{GHz}$ from a sea-based platform in the North Sea," J. Geophys. Res. Oceans, vol. 91, no. C8, pp. 9701-9708, 1986

[7] W. C. Keller, W. J. Plant, R. A. Petitt, and E. A. Terray, "Microwave backscatter from the sea: Modulation of received power and Doppler bandwidth by long waves," J. Geophys. Res. Oceans, vol. 99, no. C5, pp. 9751-9766, 1994.

[8] W. C. Keller and J. W. Wright, "Microwave scattering and the straining of wind-generated waves," Radio Sci., vol. 10, pp. 139-147, 1975.

[9] W. J. Plant, W. C. Keller, and A. Cross, "Parametric dependence of ocean wave-radar modulation transfer functions," J. Geophys. Res. Oceans, vol. 88, no. C14, pp. 9747-9756, 1983.

[10] J. Schröter, F. Feindt, W. Alpers, and W. C. Keller, "Measurement of the ocean wave-radar modulation transfer function at $4.3 \mathrm{ghz}$," $J$. Geophys. Res. Oceans, vol. 91, no. C1, pp. 923-932, 1986.

[11] D. E. Weissman and J. W. Johnson, "Measurements of ocean wave spectra and modulation transfer function with the airborne twofrequency scatterometer," J. Geophys. Res. Oceans, vol. 91, no. C2, pp. 2450-2460, 1986

[12] T. Hara and W. J. Plant, "Hydrodynamic modulation of short windwave spectra by long waves and its measurement using microwave backscatter," J. Geophys. Res. Oceans, vol. 99, no. C5, pp. 9767-9784, 1994.

[13] S. A. Ermakov, I. A. Kapustin, V. N. Kudryavtsev, I. A. Sergievskaya,
O. V. Shomina, B. Chapron, and Yu. Yu. Yurovskiy, "On the doppler frequency shifts of radar signals backscattered from the sea surface," Radiophys. Quant. Electron., vol. 57, no. 4, pp. 239-250, 2014.

[14] W. Alpers and K. Hasselmann, "The two-frequency microwave technique for measuring ocean-wave spectra from an airplane or satellite," Bound.-Layer Meteor., vol. 13, no. 1, pp. 215-230, 1978.

[15] R. Romeiser, A. Schmidt, and W. Alpers, "A three-scale composite surface model for the ocean wave-radar modulation transfer function," J. Geophys. Res. Oceans, vol. 99, no. C5, pp. 9785-9801, 1994.

[16] V. Kudryavtsev, D. Hauser, G. Caudal, and B. Chapron, "A semiempirical model of the normalized radar cross section of the sea surface, 2. Radar modulation transfer function," J. Geophys. Res. Oceans, vol. 108, C08055, doi: 10.1029/2001JC001004, 2003.

[17] J. A. Johannessen, B. Chapron, F. Collard, V. Kudryavtsev, A. Mouche, D. Akimov, and K.-F. Dagestad, "Direct ocean surface velocity measurements from space: Improved quantitative interpretation of Envisat ASAR observations," Geophys. Res. Lett., vol. 35, L22608, 2008, doi:10.1029/2008GL035709.

[18] Yu. Yu. Yurovsky, V. N. Kudryavtsev, S. A. Grodsky, and B. Chapron, "Ka-Band Dual Copolarized Empirical Model for the Sea Surface Radar Cross Section," IEEE Trans. Geosci. Remote Sens., vol. 55, no. 3, pp. 1629-1647, 2017.

[19] V. N. Kudryavtsev, B. Chapron, A. G. Myasoedov, F. Collard, and J. A. Johannessen, "On Dual Co-Polarized SAR Measurements of the Ocean Surface,” IEEE Geosci. Remote Sens. Lett., vol. 10, pp. 761-765, 2013.

[20] C. W. Fairall, E. F. Bradley, J. E. Hare, A. A. Grachev, and J. B. Edson, "Bulk Parameterization of Air Sea Fluxes: Updates and Verification for the COARE Algorithm," J. Clim., vol. 16, pp. 571-591, 2003.

[21] D. Johnson, "DIWASP, a directional wave spectra toolbox for MATLAB: User Manual," Res. Rep. WP-1601-DJ (V1.1), Centre for Water Research, University of Western Australia.

[22] A. T. Jessup, W. K. Melville, and W. C. Keller, "Breaking waves affecting microwave backscatter 1. Detection and verification," $J$. Geophys. Res. Oceans, vol. 96, pp. 20 547-20 559, 1991.

[23] W. J. Plant, "The Modulation Transfer Function: Concept and Applications," Radar Scattering from Modulated Wind Waves, pp. $155-172,1989$.

[24] P. H. Y. Lee, J. D. Barter, K. L. Beach, C. L. Hindman, B. M. Lake, H. Rungaldier, J. C. Shelton, A. B. Williams, R. Yee, and H. C. Yuen, "X band microwave backscattering from ocean waves," J. Geophys. Res. Oceans, vol. 100, pp. 2591-2612, 1995.

[25] W. J. Plant, "A model for microwave Doppler sea return at high 
incidence angles: Bragg scattering from bound, tilted waves," $J$. Geophys. Res. Oceans, vol. 102, pp. 21 131-21 146, 1997.

[26] Y. Liu, S. J. Frasier, and R. E. McIntosh, "Measurement and classification of low-grazing-angle radar sea spikes," IEEE Trans. Ant. Propagat., vol. 46, pp. 27-40, 1998.

[27] J. Seemann, M. Stresser, F. Ziemer, J. Horstmann, and L. C. Wu, "Coherent microwave radar backscatter from shoaling and breaking sea surface waves," in OCEANS 2014 - TAIPEI, April 2014, pp. 1-5.

[28] T. Lamont-Smith, T. Waseda, and C. K. Rheem, "Measurements of the doppler spectra of breaking waves," IET Radar, Sonar Navigation, vol. 1, no. 2, pp. 149-157, April 2007.

[29] A. T. Jessup, W. K. Melville, and W. C. Keller, "Breaking waves affecting microwave backscatter 2. Dependence on wind and wave conditions," J. Geophys. Res. Oceans, vol. 96, pp. 20 561-20 569, 1991.

[30] M. J. Smith, E. M. Poulter, and J. A. McGregor, "Doppler radar measurements of wave groups and breaking waves," J. Geophys. Res. Oceans, vol. 101, pp. 14269-14282, 1996.

[31] O. M. Phillips, "Spectral and statistical properties of the equilibrium range in wind-generated gravity waves," J. Fluid Mech., vol. 156, pp. $505-531,1985$.

[32] V. A. Dulov, V. Kudryavtsev, and A. N. Bolshakov, "A field study of white caps coverage and its modulations by energy containing waves," in Gas Transfer at Water Surface, Donelan M.A., Drennan W.M., Saltzman E.S., and Wanninkhof R., Eds. AGU, 2002, vol. 127, pp. 187-192.

[33] W. J. Plant, "Whitecaps in deep water," Geophys. Res. Lett., vol. 39, no. 16, 2012, L16601, doi:10.1029/2012GL052732.

[34] M. W. Hansen, V. Kudryavtsev, B. Chapron, J. A. Johannessen, F. Collard, K.-F. Dagestad, and A. A. Mouche, "Direct measurements of ocean surface velocity from space: Interpretation and validation," Remote Sens. Env., vol. 120, pp. 113-122, 2012.

[35] V. Kudryavtsev and B. Chapron, "On Growth Rate of Wind Waves: Impact of Short-Scale Breaking Modulations," J. Phys. Oceanogr., vol. 46, no. 1, pp. 349-360, 2016.

[36] V. Kudryavtsev, M. Yurovskaya, B. Chapron, F. Collard, and C. Donlon, "Sun glitter imagery of ocean surface waves. Part 1: Directional spectrum retrieval and validation," J. Geophys. Res. Oceans, vol. 122, no. 2, pp. 1369-1383, 2017.

[37] —, "Sun glitter imagery of surface waves. Part 2: Waves transformation on ocean currents," J. Geophys. Res. Oceans, vol. 122, no. 2, pp. 1384-1399, 2017.

[38] Yu. Yu. Yurovsky, V. Kudryavtsev, and B. Chapron, "Simultaneous radar and video observations of the sea surface in field conditions," in 2017 Progress in Electromagnetic Research Symposium (PIERS), St Petersburg, Russia, May 2017, in press.

[39] M. V. Yurovskaya, V. A. Dulov, B. Chapron, and V. N. Kudryavtsev, "Directional short wind wave spectra derived from the sea surface photography," J. Geophys. Res. Oceans, vol. 118, pp. 4380-4394, 2013.

[40] V. Kudryavtsev, I. Kozlov, B. Chapron, and J. A. Johannessen, "Quad-polarization sar features of ocean currents," J. Geophys. Res. Oceans, vol. 119, no. 9, pp. 6046-6065, 2014.

[41] V. Kudryavtsev, D. Hauser, G. Caudal, and B. Chapron, "A semiempirical model of the normalized radar cross-section of the sea surface 1. Background model," J. Geophys. Res. Oceans, vol. 108, C08054, doi: 10.1029/2001JC001003, 2003.

[42] V. Kudryavtsev, A. Myasoedov, B. Chapron, J. A. Johannessen, and F. Collard, "Imaging mesoscale upper ocean dynamics using synthetic aperture radar and optical data," J. Geophys. Res. Oceans, vol. 117, C04029, 2012, doi:10.1029/2011JC007492.

[43] N. Rascle, F. Nouguier, B. Chapron, A. Mouche, and A. Ponte, "Surface Roughness Changes by Finescale Current Gradients: Properties at Multiple Azimuth View Angles," J. Phys. Oceanogr., vol. 46, no. 12, pp. 3681-3694, 2016

[44] Y. Wang, H. Li, Y. Zhang, and L. Guo, "The measurement of sea surface profile with x-band coherent marine radar," Act. Oceanolog. Sinica, vol. 34, no. 9, pp. 65-70, 2015, doi:10.1007/s13131-015-0731-7.

[45] F. G. Bass, I. M. Fuks, A. I. Kalmykov, I. E. Ostrovsky, and A. D Rosenberg, "Very high frequency radiowave scattering by a disturbed sea surface. part i: Scattering from a slightly disturbed boundary, part ii: Scattering from an actual sea surface," IEEE Trans. Ant. Propagat., vol. 16 , no. 5, pp. 554-568, 1968.

[46] J. Wright, "A new model for sea clutter," IEEE Trans. Ant. Propagat., vol. 16, no. 2, pp. 217-223, 1968.

[47] F. T. Ulaby, R. K. Moore, and A. K. Fung, Microwave Remote Sensing: Active and Passive. Dedham, USA: Artech House, 1986, vol. 3, pp. 1065-2126.

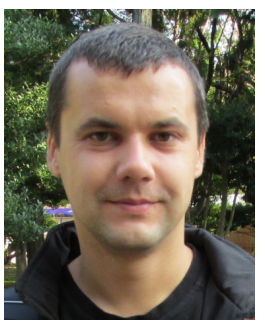

Yury Yurovsky was born in Yalta, Crimea, USSR in 1984. He received the M.S. degree in Geophysics from the Black Sea Branch of Moscow State University, Sevastopol, Ukraine in 2006. Since 2007 he works at Marine Hydrophysical Institute (MHI), Sevastopol, Russia, from where he received the $\mathrm{Ph} . \mathrm{D}$. degree in Marine physics in 2012. Currently he holds Researcher position at MHI with participating in activities at Black Sea Research platform. His work mainly includes obtaining and analysis of the data in various field experiments. His scientific interests include ocean radar and optical remote sensing, sea surface dynamics, air-sea interaction.

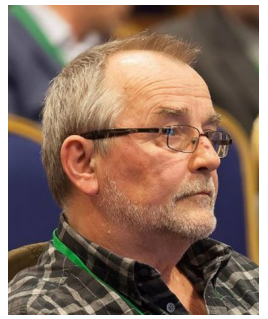

Vladimir Kudryavtsev received the Ph.D. degree and the Senior Doctorate degree in geophysics/marine physics from the Marine Hydrophysical Institute (MHI), Sevastopol, Crimea, in 1981 and 1991, respectively. In 1976, he joined MHI, where he is currently a Part-Time Principal Scientist. Since 2002, he has been a Professor at the Russian State Hydrometeorological University, St. Petersburg, where he is also the Head of the Satellite Oceanography Laboratory. From 2005 to 2013, he was with the Nansen Centre, St.Petersburg, Russia. Until July 2016, he held a part-time senior position with the Nansen Centre, Bergen, Norway. His current research interests include air-sea interaction, atmospheric and oceanic boundary layers, and radar and optical imaging of the ocean surface.

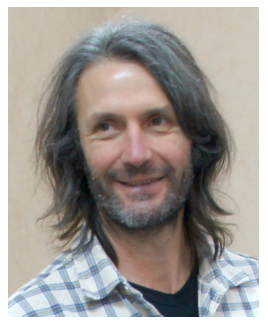

Bertrand Chapron was born in Paris, France, in 1962. He received the B.Eng. degree from the Institut National Polytechnique de Grenoble, Grenoble, France, in 1984 and the Doctorat National (Ph.D.) degree in fluid mechanics from the University of Aix-Marseille II, Marseille, France, in 1988. He spent three years as a Post-Doctoral Research Associate at the NASA/GSFC/Wallops Flight Facility, Wallops Island, VA. He has experience in applied mathematics, physical oceanography, electromagnetic waves theory, and its application to ocean remote sensing. He is currently responsible for the Oceanography from Space Laboratory, IFREMER, Plouzané, France.

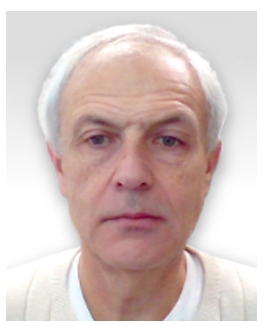

Semyon Grodsky received the Ph.D. degree in geophysics from the Marine Hydrophysical Institute (MHI), Sevastopol, Crimea, in 1986. He worked at MHI through 1999 before joining the Atmospheric and Oceanic Science Department, University of Maryland/College Park where he is currently a research scientist. His research interests include satellite remote sensing, air-sea interactions, and various aspects of ocean data analysis. 